\title{
A Temperature-Profile Method for Estimating Flow Processes in Geologic Heat Pipes
}

\author{
Jens T. Birkholzer \\ Ernest Orlando Lawrence Berkeley National Laboratory (LBNL) \\ 1 Cyclotron Road \\ Berkeley CA 94720 \\ phone 5104867134,fax 5104865686,jtbirkholzer@lbl.gov
}

\begin{abstract}
Above-boiling temperature conditions, as encountered, for example, in geothermal reservoirs and in geologic repositories for the storage of heat-producing nuclear wastes, may give rise to strongly altered liquid and gas flow processes in porous subsurface environments. The magnitude of such flow perturbation is extremely hard to measure in the field. We therefore propose a simple temperature-profile method that uses high-resolution temperature data for deriving such information. The energy that is transmitted with the vapor and water flow creates a nearly isothermal zone maintained at about the boiling temperature, referred to as a heat pipe. Characteristic features of measured temperature profiles, such as the differences in the gradients inside and outside of the heat pipe regions, are used to derive the approximate magnitude of the liquid and gas fluxes in the subsurface, for both steady-state and transient conditions.
\end{abstract}




\section{Introduction}

Thermal perturbations can have a strong impact on underground flows, in particular when the temperatures go beyond the boiling point of water and vapor is generated at significant rates. Such conditions often result in strong counterflow of liquid and gas phases and significant convective transfer of heat, commonly known as geologic "heat pipes" (e.g., Udell, 1985; Doughty and Pruess, 1990, 1992). Originally, the term "heat pipe" described engineered devices-typically hollow cylinders filled with a vaporizable liquid-that are used in many industrial applications to increase the efficiency of heat transport. Both technical and geologic heat pipes operate on the same basic principles. Since the density of vapor is much smaller than the density of liquid water, vaporization near the heat source creates a pressure buildup and causes vapor transport to cooler, more distant regions. The vapor condenses and deposits a large amount of latent heat. This condensation gives rise to an increase in saturation, thereby creating a capillary pressure gradient that drives the reflux of liquid water back to the heat source. The liquid vaporizes again and repeats the cycle. For condensate deposited above the heat source, gravity effects amplify the magnitude of reflux towards the boiling zone (Birkholzer et al., 2004).

A thorough understanding of geologic heat pipes and their effect on flow and transport processes is essential, for example, in vapor-dominated geothermal systems such as the Geysers in California (e.g., Pruess, 1985), in thermally enhanced oil recovery, and in subsurface systems affected from buried pipelines and electrical cables (Udell, 1985). Heat pipes are also expected in the vicinity of the geologic repository for nuclear waste at 
Yucca Mountain, Nevada (Pruess et al., 1990a, 1990b). Determining the magnitude of vapor-liquid counterflow in a heat pipe is relevant to the performance of this repository because the temperature and relative humidity close to the waste packages-important parameters for canister corrosion-are influenced by the relative rates of conductive and convective heat transfer. The reflux generated by heat pipes also raises concern about the potential for water seepage into the emplacement tunnels at Yucca Mountain (Birkholzer et al., 2004). Rapid preferential flow of condensate back to the heated rock zone may lead to local intrusion of water into the tunnels, even when the average temperature in the near-field rock is still above boiling (Birkholzer and Ho, 2003). Large-scale heater tests have been conducted in underground research tunnels at Yucca Mountain to assess the future repository's response to the decay heat and to determine the impact of thermal perturbation on liquid and gas flow, showing clear evidence of heat pipe behavior in the fractured porous rock (e.g., Birkholzer and Tsang, 2000; Bechtel SAIC Company, 2004a).

While knowledge about the magnitude of vapor and liquid flow is very valuable in complex thermal settings such as geothermal reservoirs and geologic repositories, it is virtually impossible to directly measure these quantities in the field. A common practice is therefore to employ numerical models calibrated to data that can be more easily measured in the field. However, the coupled flow processes are very complex, and the model parameters affecting the flow field (e.g., hydraulic conductivity) often vary over orders of magnitude in field settings. Thus, the calibration results may not always be unique, and quite often the flow estimates from numerical models are not very reliable. In 
this paper, we propose a temperature-profile method that allows us to directly determine the quantity of reflux processes in geologic heat pipes.

Temperature-profile methods have been originally employed to estimate ambient percolation fluxes in vadose environments, using measured deviations from the conduction-only geothermal gradient to evaluate the rate of convective heat transport with the percolating water (e.g, Bredehoeft and Papadopolous, 1965; Constantz et al., 2003). Using a similar approach, our proposed method utilizes the fact that the vaporliquid flow processes within a heat pipe transmit a significant amount of energy. This creates a nearly isothermal zone maintained at about the boiling temperature, with temperature gradients much smaller than those in the surrounding conduction-dominated regions. We will show in this paper that the difference between the temperature gradient within a heat pipe and the larger gradient in the above-boiling temperature region is approximately proportional to the amount of condensate reflux towards the heated zone. Application of this method requires temperature profile data in sufficient resolution, knowledge of thermal properties, and a general notion on the geometry of the heat transfer behavior (for example, linear versus radial-symmetry). Since the measurements of temperature and thermal conductivity are relatively simple and accurate in subsurface systems, the estimated reflux rates using this method are approximate, depending on the quality of the temperature data, but in general fairly reliable.

The proposed temperature-gradient method for heat pipes enables us to directly quantify the liquid and gas flow processes in a complex thermal-hydrological setting. In cases 
where numerical models exist for a given application, results from the temperatureprofile method can be used as an additional piece of evidence for model calibration and validation. Provided that temperature profiles are available at many different locations in the thermal-hydrological system, the estimated fluxes provide valuable knowledge on the heterogeneity of flow processes, allowing us to distinguish between regions of large and small reflux. In the following sections, we discuss basic features of a heat pipe using a simple example problem, present the theoretical basis for the proposed temperatureprofile method at both steady-state and transient conditions, and test the method in comparison with numerical solutions of thermally driven flow processes in porous subsurface sýstems.

\section{Conceptual Model}

\subsection{Thermal and Hydrological Conditions Influenced by Heat Pipes}

To illustrate the typical conditions inside of and near heat pipes in geologic media, we discuss an example problem with simplified radial geometry, as described in Doughty and Pruess (1992) and Pruess et al. (1999). A heat source with a constant-strength line load of $667 \mathrm{~W} / \mathrm{m}$ is placed at radius $r=0 \mathrm{~m}$ into an unsaturated porous-medium environment with the properties given in Table 1. (For average waste canisters at Yucca Mountain, this thermal power corresponds to wastes approximately 40 years old.) Initially, the flow system is at steady state with no flow processes occurring; pressure, temperature, and liquid saturation have uniform values of $P=1 \mathrm{bar}, T=18^{\circ} \mathrm{C}$, and $S_{L}=0.8$, respectively. These same values are set as fixed boundary conditions at an outer boundary far enough from the heat source so as not to affect the heat-pipe problem. Gravity is not considered. The numerical simulator TOUGH2 (Pruess et al., 1999) was 
employed to simulate the transient two-phase, two-component fluid and heat flow processes that evolve once the system starts heating up.

Figure 1a shows the simulated results in form of temperature and liquid saturation at time $t=4$ years after heating is initiated. (For comparison, temperature profiles are also given at times $t=2$ years and $t=8$ years.) Profiles of both liquid and gas fluxes are depicted in Figure $1 b$, for the same time step. Although the chosen example case is simplified, it shows all the main characteristics of a more realistic, natural heat pipe. Both the temperature and flux profiles indicate clearly that a heat pipe has developed at 4 years that stretches from about $2.2 \mathrm{~m}$ to about $3.6 \mathrm{~m}$ in radius. Temperature is nearly isothermal in this region, with a gradient much smaller than the upstream or the downstream one. Liquid saturation is zero at the inner radius of the heat pipe and slightly higher than the initial value of 0.8 at the outer radius, indicating that water boils off at one end and vapor is deposited at the other. This coincides with the significant gas flux of about $540 \mathrm{~mm} / \mathrm{yr}$ in the outward direction (positive values). The outward gas flow is almost balanced by an inward liquid flux (negative values), driven by the strong capillary pressure gradient generated from saturation differences. Note that the maximum fluxes of both gas and liquid occur close to the boiling end of the heat pipe.

The inner conduction region (between $r=0$ and $2.2 \mathrm{~m}$ ) is completely dry. With temperatures significantly above the boiling point, all liquid water has vanished. (Significant amounts of liquid water can only exist at above-boiling temperatures as a result of vapor pressure lowering in porous media with very strong capillarity. Such cases 
are not considered in this paper.) Gas flow is zero in this region because the inner boundary is closed and offers no possibility for pressure release. The outer conduction region (beyond $r=3.6 \mathrm{~m}$ ) has essentially no gas flow but some outward liquid flow. Since liquid saturation has increased above its initial value due to condensation, capillary pressure differences drive water not only back to the heat source, but, at a lesser rate, also towards the ambient-saturation regions farther away from the heat source.

Figure 1a also depicts the mass fraction of air in the gas phase. The air mass fraction is zero in both the inner conduction zone and the heat pipe region, indicating that the system is vapor dominated at temperatures above and close to boiling. Similarly, for the conditions considered, the mass fraction of air in the liquid phase is essentially zero. In the context of this paper, where the focus is on the heat and mass transfer processes within the heat pipe and the inner conduction zone, the component air can be neglected in both the liquid and gas phases, and the terms "water" and "liquid" as well as "vapor" and "gas" are used interchangeably.

In the literature, heat pipes are often described as steady-state systems, meaning that the location and intensity of the heat pipe does not vary with time (stationary heat pipes). In the steady-state case, the energy conducted from the heat source to the heat pipe region must be in equilibrium with the energy required to vaporize the refluxing water. The system depicted in Figure 1 has not yet reached a steady-state situation. As the additional temperature profiles for $t=1$ year and $t=8$ years demonstrate, the heat pipe slowly moves away from the heat source, transporting the regions of vaporization and 
condensation further outward. Thus, the energy provided by the heat source is not only needed to vaporize refluxing water, but also to change the temperature in the solid phase and to boil the resident pore water in the system as the heat pipe migrates. Because of pore water vaporization, the gas flux shown in Figure $1 \mathrm{~b}$ is slightly larger than the rate of liquid flux (i.e., more vapor is produced than provided by refluxing water). The considered example will eventually end up at steady-state conditions when the outer boundary of the model area is felt, provided that the heat load remains constant. In contrast, temporal changes in the heat load, as to be expected for the decay heats emplaced at Yucca Mountain, will continuously change the heat pipe location and intensity with time.

\subsection{Temperature-Gradient Method for Heat Pipes}

The temperature-gradient method proposed in this paper is based on simple onedimensional mass- and energy-balance formulations for a finite volume as depicted in Figure 2. We derive the governing equations for a radial-symmetric system; conversion into a system with linear heat and mass flow is straightforward. The finite volume is defined by the inner radius $r_{l}$ and the outer radius $r_{2}$, spanning a distance $\Delta r$. The radii are chosen such that the volume incorporates a small portion of rock at the boiling end of the heat pipe, with $r_{1}$ situated in the inner conduction zone and $r_{2}$ situated within the heat pipe region.

Based on the observations in Section 2.1 (Figure 1), the following mass and heat flow components enter or leave the finite volume. At $r_{l}$, across interfacial area $A_{l}=2 \pi r_{l}$, gas and liquid flow rates are zero $\left(Q_{L, 1}=Q_{G, 1}=0\right)$, while a significant amount of conductive 
heat enters the finite volume owing to the large upper-temperature gradient $\nabla T_{1}$. At $r_{2}$, across interfacial area $A_{2}=2 \pi r_{2}$, a much smaller amount of conductive heat leaves the element, since the temperature gradient $\nabla T_{2}$ is almost zero in the heat pipe region. Liquid water enters the finite volume across $A_{2}$ at mass flow rate $Q_{L, 2}$. As this water boils off, vapor is produced within the finite volume and leaves across $A_{2}$ at mass flow rate $Q_{G, 2}$. These flow processes result in convective transport of heat with the liquid phase $\left(Q_{L, 2} h_{L}\right)$ and with the gas phase $\left(Q_{G, 2} h_{G}\right)$, where $h_{L}$ is the specific enthalpy of liquid water and $h_{G}$ is the (much larger) specific enthalpy of vapor. (Note that the conceptual and mathematical formulation of the temperature-gradient method in the Appendix is developed for the more general situation where $Q_{G, 1} \neq 0$; i.e., a situation where the vapor produced in the finite volume escapes into both directions. Bidirectional vapor flow can occur in the not-uncommon case that the heat-source location coincides with an open boundary for gas flow. This situation is given, for example, at Yucca Mountain, where hot waste packages are placed into open tunnels, while a heat pipe forms in the adjacent fractured rock.)

Balancing the mass and heat flow components for the finite volume results in a set of equations for steady-state and transient heat pipes that link the magnitude of the water reflux to the temperature gradients measured at the inner and the outer radii of the element. Details on the governing equations and the mathematical derivations are given in the Appendix. 
For steady-state heat pipes, the mass flow rate $Q_{L, 2}^{S}$ of water driven back towards the boiling region in a heat pipe can be approximated as

$$
Q_{L, 2}^{S} \approx \frac{2 \pi \lambda\left(r_{1} \nabla T_{1}-r_{2} \nabla T_{2}\right)}{\left(h_{G}-h_{L}\right)}
$$

Equation (1), referred to hereafter as the quasi-steady solution, esśentially states that the net amount of energy conducted to the finite volume is equal to the energy required to vaporize the refluxing liquid water. Since the produced vapor needs to escape from the volume (no storage at steady state), the gas flow rate naturally has identical magnitude, but opposite direction to the liquid flow rate $\left(Q_{G, 2}=-Q_{L, 2}\right)$. The terms on the right-hand side of Equation (1) contain either site-specific quantities that can be easily determined from laboratory and field measurements (thermal conductivity $\lambda$, radii, temperature gradients) or known thermodynamic properties of water and vapor (specific enthalpies).

For transient heat pipes, the mass and energy balance equations include a storage term (see Appendix). As the heat pipe moves away from the heat source, resident pore water vaporizes and exits the finite volume, in addition to vapor produced from refluxing water. Also, some fraction of energy is necessary to accommodate the heating of lowertemperature regions encountered by the migrating finite volume. Given that the transient characteristics of a heat pipe can be described using a boiling front velocity $\dot{v}$, the mass flow rate $Q_{L, 2}^{T}$ in a transient heat pipe can be approximately determined by adjusting the steady-state mass flow rate $Q_{L, 2}^{S}$ using a transient flow rate correction $Q_{L, 2}^{\text {corr }}$ as follows

$$
Q_{L, 2}^{T} \approx Q_{L, 2}^{S}+Q_{L, 2}^{c o r r}
$$


with

$$
Q_{L, 2}^{c o r r} \approx 2 \bar{\pi} \dot{v}(1-\phi) \frac{\rho_{S} C_{S}\left(T_{1}-T_{2}\right)}{\left(h_{G}-h_{L}\right)}+2 \bar{\pi} \dot{r} \phi \rho_{L} S_{L, 2}
$$

Here, $\bar{r}$ is the average radius of the finite volume. The first term in Equation (3) can be determined in a straightforward manner; it contains mostly site-specific quantities that can be easily measured (porosity, average radius, grain density, grain heat capacity, temperature) or known thermodynamic properties of water and vapor (specific enthalpies). The boiling front velocity $\dot{v}$ can be estimated from temperature profiles measured at sufficient temporal resolution. The second term contains the liquid saturation $S_{L, 2}$ at location $r_{2}$ within the heat pipe. Unfortunately, it is not easy to measure this parameter in the field. We will demonstrate below that the transient flow rate corrections are quite often much smaller than the steady-state flow rates, and that transient heat pipes can then be treated as quasi-steady systems for the purpose of estimating flow. The transient contribution is often negligible because the migration of the boiling front is relatively slow and because the temperature as well as the saturation differences within the finite volume are moderate. Nevertheless, Equation (3) should always be exercised to confirm that the transient component is indeed of small importance to the flow rate results. In case $S_{L, 2}$ is not available from field measurements, this check should be done using a range of reasonable estimates for the liquid saturation at radius $r_{2}$.

The resulting quasi-steady and transient mass flow rates, as well as the transient corrections, denote the liquid flow over the entire radial-symmetric interfacial area $A_{2}$. These can be easily converted into a Darcy fluxes as follows 


$$
q_{L, 2}^{S}=\frac{Q_{L, 2}^{S}}{2 \pi r_{2} \rho_{L}} ; \quad q_{L, 2}^{T}=\frac{Q_{L, 2}^{T}}{2 \pi r_{2} \rho_{L}} ; \quad q_{L, 2}^{c o r r}=\frac{Q_{L, 2}^{c o r r}}{2 \pi r_{2} \rho_{L}}
$$

Note the convention of positive fluxes moving outward, in positive $r$-direction. Thus the liquid fluxes, directed back towards the heat source, would come out as a negative value according to this convention.

In a system with linear-geometry heat flow processes, Equations (1) and (3) can be formulated independent of the radius $r$. Eliminating radial contributions, the mass flow rate in a linear-geometry heat pipe can be approximated for quasi-steady conditions:

$$
Q_{L, 2}^{s} \approx \frac{\lambda\left(\nabla T_{1}-\nabla T_{2}\right)}{\left(h_{G}-h_{L}\right)} .
$$

For transient conditions in a linear system, the steady-state mass flow rate is adjusted using Equation (2), with the transient flow rate correction given as

$$
Q_{L, 2}^{\text {corr }} \approx \dot{v}(1-\phi) \frac{\rho_{S} C_{S}\left(T_{1}-T_{2}\right)}{\left(h_{G}-h_{L}\right)}+\dot{v} \phi \rho_{L} S_{L, 2},
$$

The mass flow rates in Equations (5) and (6) can be converted into Darcy fluxes as follows

$$
q_{L, 2}^{S}=\frac{Q_{L, 2}^{S}}{\rho_{L}} ; \quad q_{L, 2}^{T}=\frac{Q_{L, 2}^{T}}{\rho_{L}} ; \quad q_{L, 2}^{\text {corr }}=\frac{Q_{L, 2}^{\text {corr }}}{\rho_{L}}
$$




\section{Testing the Method for A Radial-Symmetric Example}

To test its potential, we apply the temperature-gradient method to the radial-symmetric example problem introduced in Section 2.1 (see simulation results in Figure 1). Let us assume that the simulated temperature profiles are measured data from a field application. The nodal points of the finite volume discretization may represent temperature sensors distributed along a borehole that measure the temperature profile in radial direction. Let us also assume that the thermal conductivity used in the example problem is known from field or laboratory measurements. We may then apply Equations (1) through (4) to determine the approximate liquid fluxes at quasi-steady and transient conditions; 'respectively, and may compare these fluxes with the simulation results given by the numerical model. Good agreement between the estimated and simulated fluxes would suggest that the temperature-gradient method works in principle.

The starting point of this process is a thorough analysis of the temperature profiles. A valuable practice is to determine the gradients between two adjacent sensors (nodal points) and to plot these together with the temperature profile. Plotting the gradients for the example problem clearly reveals the presence of a heat pipe at $t=4$ years (Figure 3 ), with a strong change of the gradient within a short distance between $r \approx 2.2 \mathrm{~m}$ and $r \approx$ $2.4 \mathrm{~m}$. We choose a finite volume with appropriate radii $r_{1}$ and $r_{2}$ near the boiling end of the heat pipe, making sure that these radii are outside of the change-of-gradient region. In other words, radius $r_{l}$ should be safely located in the inner conduction zone, with gradient changes related only to the radial geometry of the conductive heat flow processes. Radius $r_{2}$ should be safely located in the heat pipe region, where the temperature gradients are 
small and remain almost uniform with increasing radius. On the other hand, as pointed out earlier, $r_{2}$ needs to be chosen near the inner end of the heat pipe where the maximum water reflux occurs (see Figure 1). With given radii, temperature gradients, and thermal conductivity, we use Equation (1) to determine the liquid fluxes in the heat pipe under quasi-steady assumptions.

Application of Equations (2) and (3) for a transient heat pipe requires determination of the boiling front velocity $\dot{v}$. The boiling front velocity can be easily estimated from temperature profiles measured at different times. To estimate the boiling front velocity for a heat pipe at time $t$, we suggest using two additional temperature profiles measured at times $t_{a}=t-\Delta t$ and $t_{b}=t+\Delta t$. The next steps are to determine the boiling point locations $r, r_{a}$, and $r_{b}$ at times $t, t_{a}$, and $t_{b}$ (i.e., the locations of the boiling end of each heat pipe), to obtain the differences $\Delta r_{a}=r-r_{a}$ and $\Delta r_{b}=r_{b}-r$ between these locations, and to calculate two boiling front velocities $\dot{v}_{a}=\Delta r_{a} / \Delta t$ and $\dot{v}_{b}=\Delta r_{b} / \Delta t$. The resulting boiling front velocity $\dot{v}$ at time $t$ is then derived as the arithmetic average of the two values $\dot{v}_{a}$ and $\dot{v}_{b}$. Note that $\Delta t$ should be chosen small enough such that the boiling front velocities at $t_{a}$ and $t_{b}$ are similar to that at time t. On the other hand, $\Delta t$ needs to be large enough such that the three temperature profiles have measurably different boiling point locations.

The thermodynamic properties of water and steam in Equations (1) through (7) are a function of temperature and pressure. Since the finite volume defined for the temperature-gradient method is at the boiling end of the heat pipe, the specific enthalpies 
of water and vapor as well as the density of water should ideally be determined at the boiling point of water, which varies with pressure. Pressure, in turn, is affected by vaporization, which generally gives rise to some pressure buildup in the inner conduction zone and, to a lesser extent, in the heat pipe region. However, the temperature and pressure dependence of the relevant thermodynamic properties is not significant and can be neglected in most practical applications. For example, a boiling point increase from $T=99.6^{\circ} \mathrm{C}$ to $T=111.4^{\circ} \mathrm{C}$, corresponding to a (quite significant) pressure increase from $P=1$ bar to $P=1.5$ bar, would lead to a less than $2 \%$ decrease in the net sum of the specific enthalpies $\left(h_{G}-h_{L}\right)$ and a less than $1 \%$ decrease in water density $\rho_{L}$. These differences are much smaller than the uncertainties associated with the estimated liquid fluxes, which may stem from measurement inaccuracies, data noise, heterogeneity, and insufficient sensor resolution (see example in Section 4.3). All calculations in this paper are performed with the thermodynamic properties determined at a pressure of $1 \mathrm{bar}$ and a boiling point temperature of $T=99.6^{\circ} \mathrm{C}$ (see Table 1 ). When applying the temperaturegradient method, the inaccuracy introduced by this assumption can easily be evaluated by analyzing the pressure conditions. This is trivial for cases in which gas pressure measurements are available. Otherwise, the boiling temperature-and the corresponding gas pressure-can be estimated from the temperature measurements at the boiling end of the heat pipe. The temperature profile in Figure 3, for example, shows a temperature of about $105^{\circ} \mathrm{C}$ at this location, indicating that the local vapor pressure should be about 1.2 bar. 
Figure 4 compares the liquid fluxes obtained from the temperature-gradient method with those from the simulation model. Fluxes were estimated using the quasi-steady and the transient solutions given in Section 2-Equation (1) and Equations (2), (3), respectively. Results are presented for the temperature profile given in Figure 3 (at $t=4$ years) as well as for two additional times at $t=2$ years and $t=8$ years. The plot shows excellent agreement between the estimated fluxes and the simulated results, for all three times. The quasi-steady fluxes are almost identical to the transient fluxes, indicating that the transient flux correction is negligible. Hence, the flux estimates obtained from the quasisteady assumption provide sufficiently accurate heat-pipe fluxes for this first example case; application of the more complex transient solution is not necessary.

Table B1 in Appendix B gives more detail on how the estimated fluxes have been derived in this example case. The table lists the chosen radii $r_{l}$ and $r_{2}$ at both sides of the boiling end of the heat pipe and gives the gradients measured at those locations. The observed trend of liquid flux decreasing as the heat pipe gradually moves away from the heater is reflected in the measured temperature gradients that reduce with time and increasing radius. Note that the radii and gradients given in Table B1 are those for the "bestestimate" finite volume, with $r_{l}$ being the first radius that is safely located in the inner conduction zone. To demonstrate that the flux calculation is not sensitive to the choice of the $r_{1}$ (as long as $r_{1}$ is situated in the inner conduction zone), we have determined additional flux estimates using the next four upstream gradients, plotted as small green symbols in Figure 4. The results are virtually identical to those obtained for the "bestestimate" finite volume. 
Table B1 also provides supporting information on the transient flux-correction terms. The boiling front velocity required for estimating $Q_{L, 2}^{c o r r}$ is determined using $\Delta t=0.25$ years, meaning that, for example, the approximate progress of the boiling front at $t=4$ years is evaluated using additional temperature profiles at times $t_{a}=3.75$ years and $t_{b}=4.25$ years. The boiling front migrates with small velocities of about $0.4 \mathrm{~m}$ per year (at 2 years) down to about $0.2 \mathrm{~m}$ per year (at 8 years). Such velocities are small and do not allow for a significant transient contribution to the estimated fluxes. Table $\mathrm{Bl}$ gives the transient flux corrections separately for the temperature-dependent term and the saturationdependent term. It turns out that the transient contributions are indeed very small (on the order of a few millimeter per year), similar in magnitude to the differences between the estimated quasi-steady fluxes and the simulated results. Consequently, these transient contributions can be safely neglected in this first example case.

\section{Two-Dimensional Test Cases with Gravity-Driven Flow}

\subsection{Base Case}

In this section, the temperature-gradient method is applied to two-dimensional test cases more representative of the horizontal-tunnel emplacement design envisioned for the geologic repository at Yucca Mountain. The first test case, referred to hereafter as base case, is defined as follows. A heat source with a constant-strength line load is placed into a horizontal tunnel located in the center $(x=0 \mathrm{~m}$ and $\mathrm{z}=0 \mathrm{~m})$ of a vertical domain of 200 $\times 200 \mathrm{~m}^{2}$ extent. The axis of the heater and the tunnel axis are orthogonal to the vertical domain. The tunnel radius is $2.75 \mathrm{~m}$, identical to the future emplacement drifts planned at 
the Yucca Mountain repository. The thermal-hydrological properties in the porous medium surrounding the tunnel, as well as the initial and boundary conditions, are identical to the radial-symmetric example introduced in Section 2.1 , with the exceptions (1) that gravity is considered as a driving force for flow, (2) that the initial saturation of the subsurface is set to 0.6 instead of 0.8 , and (3) that the heater power is $1,095 \mathrm{~W} / \mathrm{m}$ instead of $667 \mathrm{~W} / \mathrm{m}$. The initial saturation was reduced in this example case to avoid unrealistically high gravity-driven percolation flux at ambient conditions. The selected heater power is identical to the average line load generated by the nine floor heaters in the Drift Scale Test, a large-scale underground heater test currently being conducted at the geologic repository for nuclear waste at Yucca Mountain (Datta et al., 2004).

The numerical simulator TOUGH2 (Pruess et al., 1999) was employed to determine the thermal-hydrological flow processes in the model domain. The open tunnel was modeled as a gas-filled, zero-capillarity medium with a thermal conductivity of $10.6 \mathrm{~W} /(\mathrm{m}-\mathrm{K})$. This large thermal conductivity approximates the effective radiative heat transfer that occurs between the heat source located in the center of the tunnel and the tunnel walls. Initially, the flow system is at steady state, with a gravity-driven percolation flux of about $115 \mathrm{~mm} / \mathrm{yr}$ in most of the vertical domain (except for the immediate tunnel vicinity). The vertical flow field is disturbed in the immediate tunnel vicinity because the tunnel opening acts as a capillary barrier, diverting the downward percolation flux sideways around the tunnel. 
Figure 5 shows a close-up view of the two-dimensional temperature and saturation fields together with liquid flux vectors at $t=4$ years. The flow system has heated up considerably to maximum temperatures of more than $160^{\circ} \mathrm{C}$ at the tunnel wall, and a dry conduction-dominated zone has evolved in the porous medium extending a few meters away from the tunnel. While the temperature field appears radial-symmetric-as the heat transfer is conduction dominated-the saturation and flux fields show distinct differences between the regions above and below the heat source. These differences result from gravitational forces. Vapor that condenses above the heat source is driven back to the boiling zone by the combined impact of capillarity and gravity. (A significant fraction of the water also diverts in the lateral direction, around the boiling zone.) As the refluxing water encounters the boiling zone, it vaporizes again and repeats the cycle, thereby enhancing the intensity of the heat pipe flows above the heater. In contrast, condensate below the heater is exposed to counteracting forces as capillarity pulls upward and gravity pulls downward. While there is a net upward flow of water just below the boiling front (because of capillary forces dominating gravity forces), a considerable fraction of the condensate drains off and is thus not available to drive the heat pipe. As a result, there are higher saturations and stronger reflux processes above the heater than below. This phenomenon should be detectable by the temperature-gradient method proposed in this paper.

Similar to Section 3, we apply the temperature-gradient method to the simulated temperature profiles, estimate the heat-pipe fluxes from the gradients, and compare the estimated fluxes to the simulated fluxes to test the accuracy of the method. First, 
however, we need to make sure that the heat transfer processes are approximately onedimensional, as the method requires. In addition, the boreholes for temperature measurements need to be oriented parallel to the direction of the main heat flow. These requirements are met in our test case, since both the temperature field and the nodal points of the finite volume discretization (representing temperature sensors) are approximately radial-symmetric (see Figure 5a). Note that the liquid fluxes depicted in Figure $5 \mathrm{~b}$ are not radial-symmetric. The temperature-gradient method provides the radial flux components in such cases, i.e., the flux components that are driven back to the boiling zone and affect the temperature profiles. Tangential fluxes do not contribute to heat pipe processes and are thus not detectable by gradient changes.

Three boreholes have been selected for the flux analysis, the first vertically up, the second vertically down, and the third horizontally in the positive $\mathrm{x}$-direction (Figure 5a). Each borehole starts at the tunnel wall at a radial distance of $2.75 \mathrm{~m}$ measured from the center of the tunnel. The vertical boreholes are parallel to the direction of flow. In contrast, fluxes have a horizontal and a vertical component for the horizontal borehole. Figure 6 shows the simulated temperatures, liquid saturations, and liquid fluxes along the three boreholes at $t=4$ years as a function of distance from the center of the tunnel. For better comparison of the heat-pipe intensity, fluxes are plotted following the convention that positive values indicate flow away from the heat source and negative values indicate flow towards the heat source. (Thus, negative fluxes above the heater flow downwards; negative fluxes below the heater flow upward). The strongest reflux occurs above the heater, where capillarity and gravity create a maximum downward flux of $-430 \mathrm{~mm} / \mathrm{yr}$ 
(Figure $6 \mathrm{~b}$ ). The reflux towards the boiling zone is significantly smaller below the heater, at $-235 \mathrm{~mm} / \mathrm{yr}$, where gravity works against capillarity. The horizontal reflux component along the third borehole is somewhere in between the two vertical ones, at $-326 \mathrm{~mm} / \mathrm{yr}$. The differences in the reflux magnitude between the three boreholes are clearly reflected in the temperature profiles and saturation profiles. The heat-pipe signatures, i.e., the differences in gradients at the boiling zone and the extent of the nearly isothermal heatpipe zone, are strongest for the vertical profile above the tunnel. Also note the different location of the boiling and the dryout zones, indicating that the outward migration of the boiling front below the tunnel is faster than above.

In Figure 7, the flux results from the temperature-profile method are plotted in comparison with the simulated fluxes for the three boreholes and three selected times at $t$ $=2,4$, and 8 years. There is excellent agreement between the transient flux estimates and the simulated fluxes. Thus, the proposed temperature-gradient method works well not only for the simple radial-symmetric example of Section 3, but also for cases with more realistic two-dimensional heat and mass flow processes. The quasi-steady results are reasonably close, but tend to overestimate the magnitude of the simulated fluxes by a few percent. In all practical field applications, such percentages are smaller than the expected margin of error associated with the temperature-gradient method (see example in Section 4.3). Thus, while the transient flux corrections are more important than in the radialsymmetric example case presented in Section 3, they are still rather insignificant compared to the overall fluxes and can be neglected without much loss in accuracy. Note 
that Table B2 in Appendix B gives additional details on the temperature-gradient calculations conducted to derive the flux estimates in Figure 7.

\subsection{Sensitivity Case with Increased Transient Contribution}

We present a second two-dimensional example to evaluate the accuracy of the temperature-gradient method for conditions where the transient contribution is more important. The base case is modified by (1) increasing the heat load to $1,450 \mathrm{~W} / \mathrm{m}$ (a value representative of the average initial thermal output of Yucca Mountain waste canisters before the waste starts decaying), and (2) increasing the porosity of the porous medium from $\phi=0.1$ to $\phi=0.4$. The first modification increases the boiling front velocity, which affects both the temperature-dependent and the saturation-dependent flux correction terms in Equation (3). The second modification affects only the saturationdependent term; the larger porosity increases the amount of pore water that is initially present in the flow system and that needs to boil off as the boiling front migrates. (Note that the emplacement strategy for the Yucca Mountain repository calls for a 50-year period of forced ventilation in the tunnels, which is expected to reduce the heat load imposed on the tunnel walls by at least $70 \%$. The effect of forced ventilation is not considered in this example case.)

Figure 8 depicts the quasi-steady and transient flux estimates from the temperaturegradient method together with the simulation results. Additional details on the flux calculation are given in Table B3. The fluxes calculated from the quasi-steady heat-pipe assumption are not very accurate. They are consistently much larger in magnitude (more 
negative) than the simulated results, indicating that a noticeable fraction of energy is used for heating the system and vaporizing pore water as the heat pipe migrates. The difference between the quasi-steady flux estimates and the simulated fluxes is as much as about $100 \mathrm{~mm} / \mathrm{yr}$, or up to $30 \%$ of the overall flux. Such differences are significant, even in light of the various uncertainties in the flux estimates, and should not be disregarded. The transient fluxes, on the other hand, are in very good agreement with the simulated target values. In other words, the transient effects can be accurately accounted for by adjusting the quasi-steady fluxes using the transient flux correction terms defined in Equation (3).

The above assessment clearly demonstrates that the approach for calculating transient fluxes works very well in theory. In praxis, however, the transient contribution may be hard to quantify in a precise manner. While calculation of the temperature-dependent flux correction is straightforward, because the temperature values $T_{1}$ and $T_{2}$ can be easily determined from the temperature profile, calculation of the saturation-dependent flux corrections requires knowledge of the liquid saturation values $S_{L, 2}$, which may not be measured in the field. Unfortunately, as the respective results in Table B3 suggest, the saturation-dependent flux correction is significantly more important than the temperaturedependent flux correction.

In light of this discussion, we suggest the following approach for the temperaturegradient method in heat pipe systems with important transient contributions. In the trivial case that saturation has been measured together with the temperature profiles-e.g., by 
having combined neutron logging and temperature measurements in boreholes (Bechtel SAIC Company, 2004b) - the transient fluxes should be calculated using the measured saturation data. Otherwise, there are two options. The first option is to simply go with the quasi-steady flux estimates (or to use the quasi-steady estimates corrected for the temperature-dependent term), knowing that these provide upper-bound values for the correct transient results. Using upper-bound values for the water reflux towards emplacement tunnels is conservative with respect to the overall performance of the geologic repository at Yucca Mountain, because this will overestimate the potential of water seeping into the tunnels and contacting waste packages. The second option is to evaluate the transient flux correction using a range of reasonable values for the liquid saturation $S_{L, 2}$. This range can be based on educated guesses, on analytical estimates (linking, for example, pressure buildup, temperature, and saturation), or on a complementary numerical study. In porous media, the range of reasonable saturation values is limited because the resulting saturations at radius $r_{2}$ are generally much smaller than the initial saturation. The simulated saturation values given in Tables B1, B2, and $\mathrm{B} 3$, for example, range from 0.09 to 0.22 , compared with the initial saturation of 0.8 . We prefer the second option over the first one because it provides approximate information on the importance of the transient component in a given heat-pipe application.

\subsection{Sensitivity Case with Heterogeneity}

In field studies, temperature measurements will be affected by measurement inaccuracies and effects of heterogeneity. To test the potential of our method in such conditions, we apply the temperature-gradient method to an additional simulation example featuring a spatially variable thermal conductivity field. Except for this modification, the example 
case is identical to the base case analyzed in Section 4.1. Thermal conductivity is chosen as the heterogeneous parameter because it has a direct impact on the temperature data used for the temperature-gradient method. An uncorrelated random field of thermal conductivity values was generated and mapped to the grid elements in the model domain, assuming a normal probability distribution with a mean thermal conductivity of $2.0 \mathrm{~W} /(\mathrm{m}-\mathrm{K})$ and a standard deviation of $0.2 \mathrm{~W} /(\mathrm{m}-\mathrm{K})$. This level of heterogeneity is representative of the thermal conductivity variability observed at Yucca Mountain (Bechtel SAIC Company, 2004c).

Figure 9.shows the simulated temperature and gradient results along the two vertical and the horizontal profiles at $t=2$ years, 4 years, and 8 years, together with the spatial distibution of the thermal conductivity values along the selected borehole. Circular symbols indicate the location of "temperature sensors"; i.e., the center nodes of the finite volume discretization. For comparison, the respective temperature results of the homogenous base case are depicted as dashed lines. The temperature profiles of the heterogeneous case appear very smooth, a result of the forgiving nature of heat conduction, and the differences between homogeneous and heterogeneous seem quite small. The gradient profiles, on the other hand, show clear indication of the effect of heterogeneity, which will challenge the accuracy of the flux estimates from the temperature-gradient method. Discrepancies between the heterogeneous temperature results and the homogeneous background profiles are directly correlated to the local thermal conductivity, with smaller conductivities creating gradients larger in magnitude (more negative). 
When applying the temperature-gradient method to the heterogeneous simulation results, we have to assume that the specific spatial distribution of thermal conductivity along the selected boreholes is unknown, as it would be in field applications. Thus, we use the average thermal conductivity value of $2.0 \mathrm{~W} /(\mathrm{m}-\mathrm{K})$ in our flux evaluation. As in the homogeneous cases, the first step is to visually define the "best" finite volume for the flux calculation at the boiling end of the observed heat pipes. However, the noise in the temperature data makes the determination of radii and the choice of gradients somewhat subjective and arbitrary in the heterogeneous case. As Figure 9 suggests, the smaller gradients $\nabla T_{2}$ are usually less problematic, with relatively small gradient changes within the heat-pipe region. The upper gradients $\nabla T_{1}$, on the other hand, exhibit considerable zigzag behavior. In such cases, the resulting flux estimates would be strongly affected by the subjective selection of radius $r_{l}$ and upper gradient $\nabla T_{1}$. To evaluate the uncertainty introduced by data noise, we generally recommend using more than one radius $r_{1}$ and gradient $\nabla T_{1}$ for the calculation of fluxes. The radii $r_{1}$ of each profile in Figure 9 , for example, have been defined by choosing the first upper gradient location clearly outside of the change-of-gradient region at the boiling end of the heat-pipe zone. The temperature-gradient method is applied to this radius and gradient, plus the next four radii and gradients in the upstream direction, and the average flux is then used as the "bestestimate" result. The range of fluxes covered by the individual flux estimates is indicative of the degree of uncertainty of the temperature-gradient method caused by heterogeneity.

Figure 10 shows the calculated flux estimates from the temperature gradient method for the three selected boreholes and three times. Solid symbols give the average fluxes over 
five upstream locations and gradients, calculated from the transient heat-pipe solution. Error bars indicate the range covered by the five individual fluxes. Again, the flux convention is that negative flux values denote flow towards the heat source. The simulated "correct" fluxes are depicted as dashed lines. For most of the heat pipes analyzed in Figure 10, the average flux estimates provide a good approximation of the simulated target values. In other words, the temperature-gradient method delivers reasonably accurate flux results even for a simulation case with considerable heterogeneity in a key thermal parameter, the details of which are assumed to be unknown in the application of the method.

The largest flux discrepancy occurs for the horizontal borehole at 4 years, where the average as well as all five individual flux estimates are smaller than the simulated result by about 30 to $60 \mathrm{~mm} / \mathrm{yr}$. This is clearly related to the prominent cluster of above-average thermal conductivity values just inward of the finite volume in Figure 9c. As a result, all five temperature gradients obtained for the flux calculation are small compared to those expected from a homogeneous medium with average thermal conductivity. Hence, one important source of uncertainty in the temperature-gradient method stems from the fact that the chosen thermal conductivity value may not be representative of the conditions where the gradients are measured. Compared to other thermal-hydrological properties of subsurface soils such as permeability, however, the spatial variability of thermal conductivity is usually fairly small in porous media applications, with variability coefficients similar to those considered in this example case. Note that the average flux estimates differ from the simulation results by less than $3 \%$ for the borehole facing 
vertically up, by less than $7 \%$ for the borehole facing vertically down, and by less than $13 \%$ for the horizontal borehole. This level of accuracy would be reasonably good for any kind of field data, but is especially impressive considering the specific difficulties and uncertainties in estimating underground fluxes in the field. Any type of measurement that can provide flux data within an uncertainty range of, say, a factor of two or three is certainly a valuable tool for the analysis of subsurface flow processes.

The error bars in Figure 10 give an indication of the uncertainty range of the flux estimates stemming from the variability of the five upstream temperature gradients. In our example, this variability is a result of heterogeneity in thermal conductivity; in field applications, some additional noise may be caused by measurement errors or by heterogeneity in other relevant flow and transport properties. Over all three boreholes and times, the error related to data noise bars ranges from about $10 \%$ to about $20 \%$ of the average fluxes. Again, this level of uncertainty is very reasonable considering the inherent uncertainty involved in measuring underground flows.

\section{Summary and Conclusions}

Evaluating the magnitude of flux perturbation in superheated subsurface systems can be a challenging task, in part because the direct in situ measurement of such quantities is virtually impossible. Flux perturbations are particularly strong in geologic heat pipes, where vapor is transported away from the heat source as condensate water flows back towards the heat source, thereby creating a continuous re-circulation of vapor and water at significant rates. The energy transported with these fluxes leaves a distinct signature in temperature profiles. We propose a temperature-gradient method for deriving the flux 
perturbation occurring in geologic heat pipes using characteristic features of temperature profiles. The method requires temperature profile data with sufficient resolution, knowledge of thermal properties, and a general idea on the geometry of the heat transfer conditions. Field measurements of temperature are relatively simple and generally fairly accurate in subsurface systems.

The theoretical framework for the temperature-gradient method is presented for two alternative heat transfer scenarios in a porous-medium environment, one assuming radial transport from a line-load heat source, the other assuming linear transport from an areaload heat source. Differences between the temperature gradients measured at both sides of the boiling end of a heat pipe are used to estimate the amount of energy available to vaporize water. For stationary heat pipes, the amount of energy is proportional to the liquid reflux in the heat pipe. For transient heat pipes, some fraction of the supplied energy is used to change the temperature and to boil the resident pore water of downstream regions encountered when the heat pipe moves away from the heat source.

The temperature-gradient method was formulated for both quasi-steady conditions (stationary heat pipes) and transient heat conditions (moving heat pipes). Transient flux correction terms have been developed in Equations (3) and (5) that adjust the quasisteady fluxes for the impact of transient effects. Exercising these transient equations requires knowledge about the velocity of the heat pipe moving away from the heat source. To determine this velocity, we suggest using temperature profiles measured at different times. The transient equations also require some knowledge of the liquid 
saturation at the boiling end of the heat pipe. If saturation has not been measured in a given field application, the transient contribution should be approximated using a range of reasonable saturation estimates.

The proposed method was tested in comparison with various one-dimensional and twodimensional example cases, for which model simulations were conducted to provide simulated temperature and flux results. The temperature results were fed as "measured data" input to the temperature-gradient method. It turned out that the fluxes estimated from applying the temperature-gradient method to these "measured data" were in excellent agreement with the simulated fluxes, demonstrating that the temperaturegradient method works in principle. The flux comparison also suggested that the transient contribution for moving heat pipes-i.e., the energy necessary to change the temperatures and to vaporize resident pore water-is often relatively small and may be safely neglected in many applications. In such cases, the fluxes estimated under quasi-steady assumptions are reasonably close to the simulated fluxes.

One of the two-dimensional example cases was simulated assuming a spatially variable thermal conductivity field. The spatial variability introduces two sources of uncertainty that are typical for field applications. The first type of uncertainty stems from the fact that the average thermal conductivity value used for the temperature-gradient analysis may be different from the local thermal conductivity at the boiling end of the heat pipe. The second uncertainty contribution is a result of data noise in the measured gradients, which leads to variability in the estimated fluxes. To evaluate this second type of uncertainty, 
we should generally derive flux estimates based on the evaluation of more than one upper heat pipe gradient. In our example case, both sources of uncertainty turned out to be reasonably small, on the order of less than $20 \%$ of the overall fluxes.

Our example applications suggest that the proposed method offers a promising approach for quantifying liquid and gas flow processes in complex thermal-hydrological settings. We are currently working on field applications, such as the analysis of data from the Drift Scale Test at Yucca Mountain, a large-scale underground heater test with high-resolution temperature data in both space and time. The estimated fluxes for the Drift Scale Test will provide an additional piece of evidence for the calibration and validation of numerical simulation models used for the prediction of the future thermal-hydrological conditions at Yucca Mountain.

\section{Appendix A: Governing Equations}

The temperature-gradient method is based on the formulation of basic mass and energy conservation equations for the radial-symmetric finite volume depicted in Figure 2. The finite volume is defined at the boiling end of the heat pipe, with radius $r_{I}$ just within the inner conduction zone and radius $r_{2}$ just within the heat pipe region. Temperature and liquid saturation are given by $T_{1}$ and $S_{L, 1}$ at radius $r_{1}$ and $T_{2}$ and $S_{L, 2}$ at radius $r_{2}$, respectively. According to Figure 2, the inner conduction zone is completely dry in porous media so that $S_{L I}$ can be set to zero. The temperature gradients are $\nabla T_{1}$ and $\nabla T_{2}$. The unknown mass flow rate of water in the heat pipe is $Q_{L, 2}$ at radius $r_{2}$. There is no liquid flow at the inner radius $r_{l}\left(Q_{L, I}=0\right)$. 
The basic mass conservation equation for two-phase flow of liquid and gas in radial geometry is given as

$$
2 \pi r \phi \frac{\partial\left(S_{L} \rho_{L}+S_{G} \rho_{G}\right)}{\partial t}+\frac{\partial\left(Q_{L}+Q_{G}\right)}{\partial r}=0
$$

where the first term represents the change in the stored mass in liquid and gas phases, and the second term represents the liquid and gas flux into and out of the control volume. Only the water component is considered in Equation (A1), as the mass fraction of air can be neglected in both liquid and gas phases. The basic energy conservation equation in radial geometry is

$$
\begin{gathered}
2 \pi r(1-\phi) \rho_{S} C_{S} \frac{\partial T}{\partial t}+2 \pi r \phi \frac{\partial\left(S_{L} \rho_{L} u_{L}+S_{G} \rho_{G} u_{G}\right)}{\partial t}+\frac{\partial\left(h_{L} Q_{L}+h_{G} Q_{G}\right)}{\partial r} \\
-\frac{\partial}{\partial r} 2 \pi r \lambda \frac{\partial T}{\partial r}=0
\end{gathered}
$$

Here, the first two terms denote the stored energy in solid, liquid, and gas phases. The third term is the convective transport of energy, which consists of liquid and gas phase contributions. The last term is the conductive transport of heat. We approximate the differential terms in Equations (A1) and (A2) using first-order derivatives and apply the resulting conservation equations to the finite volume in Figure 2. Per definition, the liquid and gas phase saturations sum to unity. It can be easily shown, then, that a change in liquid saturation corresponds to the opposite change in gas saturation, so that $\Delta S_{G}=-\Delta S_{L}$. While there is a significant decrease in the temperature gradients within the finite volume, the changes in absolute temperature are fairly small. As a result, we can neglect the temperature-dependence of liquid and gas thermodynamic properties (density, specific enthalpy, internal energy), and may consider $\rho_{L}, \rho_{G} u_{L}, u_{G}, h_{L}$, and $h_{G}$ to be 
independent of time $t$ and radius $r$. Furthermore, we assume a uniform thermal conductivity $\lambda$ and introduce the temperature gradient $\nabla T=\Delta T / \Delta r$. The mass conservation equation becomes

$$
2 \pi r \phi \frac{\left(\rho_{L}-\rho_{G}\right) \Delta S_{L}}{\Delta t}+\frac{\Delta\left(Q_{L}+Q_{G}\right)}{\Delta r} \approx 0
$$

The energy conservation equation is

$$
\begin{gathered}
2 \pi r(1-\phi) \rho_{S} C_{S} \frac{\Delta T}{\Delta t}+2 \pi r \phi \frac{\left(\rho_{L} u_{L}-\rho_{G} u_{G}\right) \Delta S_{L}}{\Delta t}+\frac{h_{L} \Delta Q_{L}+h_{G} \Delta Q_{G}}{\Delta r} \\
-2 \pi \lambda \frac{\Delta(r \nabla T)}{\Delta r} \approx 0 .
\end{gathered}
$$

Let us first analyze the case of a stationary heat pipe. As the time derivative is zero, Equation (A3) reduces to $\Delta Q_{L}=-\Delta Q_{G}$, stating that the net liquid and gas fluxes have identical magnitude, but opposite direction. This relationship is used to eliminate $\Delta Q_{G}$ from Equation (A4). With minor rearrangements, Equation (A4) becomes

$$
-\frac{\Delta Q_{L}\left(h_{G}-h_{L}\right)}{\Delta r}-2 \pi \lambda \frac{r_{2} \nabla T_{2}-r_{1} \nabla T_{1}}{\Delta r} \approx 0
$$

which can be easily solved for $\Delta Q_{L}$. With $\Delta Q_{L}=Q_{L, 2}-Q_{L, I}$ and $Q_{L, I}=0$, the mass flow rate of water at radius $r_{2}$ for stationary heat pipes is given as (see Equation (1))

$$
Q_{L, 2}^{S} \approx \frac{2 \pi \lambda\left(r_{1} \nabla T_{1}-r_{2} \nabla T_{2}\right)}{\left(h_{G}-h_{L}\right)} .
$$

Superscript $S$ denotes that the flow rate $Q_{L, 2}^{S}$ is estimated for quasi-steady heat pipes. 
For transient heat pipes, the finite volume defined at the boiling end moves in an outward direction with velocity $\dot{v}$, which can be measured in the field. It is convenient to substitute all first-order time-derivatives of variable $t$ with first-order space-derivatives of variable $r$, using $\Delta t=\Delta r / \dot{v}$. After performing this substitution in both mass and energy conservation equations, Equation (A3) is solved for the gas flow rate:

$$
\Delta Q_{G} \approx-\Delta Q_{L}-2 \pi r \phi r \dot{v} \frac{\left(\rho_{L}-\rho_{G}\right) \Delta S_{L}}{\Delta r} \approx 0
$$

and the result is inserted into Equation (A4) to eliminate the unknown $\Delta Q_{G}$

$$
\begin{gathered}
2 \pi r(1-\phi) \rho_{S} C_{S} \dot{v} \frac{\Delta T}{\Delta r}+2 \pi r \phi \dot{v} \frac{\left(\rho_{L} u_{L}-\rho_{G} u_{G}\right) \Delta S_{L}}{\Delta r}-\frac{\Delta Q_{L}\left(h_{G}-h_{L}\right)}{\Delta r} \\
-h_{G} 2 \pi r \phi \dot{v} \frac{\left(\rho_{L}-\rho_{G}\right) \Delta S_{L}}{\Delta r}-2 \pi \lambda \frac{\Delta(r \nabla T)}{\Delta r} \approx 0 .
\end{gathered}
$$

At this point, Equation (A8) contains only derivatives in space, not in time. As mentioned before, we may neglect the temperature-dependence of liquid and gas thermodynamic properties within the finite volume. Since liquid water is approximately incompressible, we can also replace internal energy $u_{L}$ with specific enthalpy $h_{L}$. Further rearrangements give

$$
\begin{gathered}
2 \pi \dot{v}(1-\phi) \rho_{S} C_{S} \frac{\Delta T}{\Delta r}-2 \pi \dot{v} \phi \rho_{L}\left(h_{G}-h_{L}\right) \frac{\Delta S_{L}}{\Delta r}-2 \pi \dot{v} \phi \rho_{G}\left(u_{G}-h_{G}\right) \frac{\Delta S_{L}}{\Delta r} \\
-\frac{\Delta Q_{L}\left(h_{G}-h_{L}\right)}{\Delta r}-2 \pi \lambda \frac{\Delta(r \nabla T)}{\Delta r} \approx 0 .
\end{gathered}
$$

The first velocity-dependent term in Equation (A9) accounts for the energy required for changing the temperature in the solid phase as the finite volume moves outward with velocity $\dot{v}$. The second term comprises the energy needed for boiling of resident pore 
water in the moving finite volume. The third term, related to energy changes associated with volumetric changes, can be eliminated, since it is several orders of magnitude smaller than the first two terms. This is because the gas density $\rho_{G}$ in the third term is much smaller than the solid- and liquid-phase densities occurring in the other terms. Also, internal energy $u_{G}$ and specific enthalpy $h_{G}$ have approximately equal magnitude.

The next step is to approximate the spatial variable $r$ in Equation (A9) using the average radius of the finite volume (i.e, $\bar{r}=\left(r_{1}+r_{2}\right) / 2$ ). We also replace $\Delta T$ and $\Delta S_{L}$ with the respective temperature and saturation differences between both ends of the finite volume $\left(\Delta T=T_{2}-T_{1}\right.$ and $\Delta S_{L}=S_{L, 2}-S_{L, 1}$, with $\left.S_{L, 1}=0\right)$. Finally, as done before with the quasi-steady solution, we use $Q_{L, 2}$ instead of $\Delta Q_{L}$ and solve Equation (A9) for the unknown mass flow rate of water in transient heat pipes:

$$
Q_{L, 2}^{T} \approx \frac{2 \pi \lambda\left(r_{I} \nabla T_{I}-r_{2} \nabla T_{2}\right)}{\left(h_{G}-h_{L}\right)}+2 \pi \bar{r} \dot{v}(l-\phi) \frac{\rho_{S} C_{S}\left(T_{1}-T_{2}\right)}{\left(h_{G}-h_{L}\right)}+2 \pi \bar{r} \dot{v} \phi \rho_{L} S_{L, 2} .
$$

Superscript $T$ denotes that the flow rate $Q_{L, 2}^{T}$ is for transient heat pipes. The first term in Equation (A10) is identical to the quasi-steady mass flow rate $Q_{L, 2}^{S}$ defined in Equation (A6). The second term accounts for the storage contribution to be considered in transient heat pipes. We may refer to this storage contribution as the transient flow rate correction $Q_{L, 2}^{\text {corr }}$, such that

$$
Q_{L, 2}^{T}=Q_{L, 2}^{S}+Q_{L, 2}^{c o r r}
$$


$Q_{L, 2}^{\text {corr }}$ comprises contributions for temperature-related energy changes (Term 1) and saturation-related energy changes (Term 2) as follows:

$$
Q_{L, 2}^{\text {corr }}=2 \pi \bar{r} \dot{v}(1-\phi) \frac{\rho_{S} C_{S}\left(T_{1}-T_{2}\right)}{\left(h_{G}-h_{L}\right)}+2 \pi \bar{r} \dot{v} \phi \rho_{L} S_{L, 2} .
$$




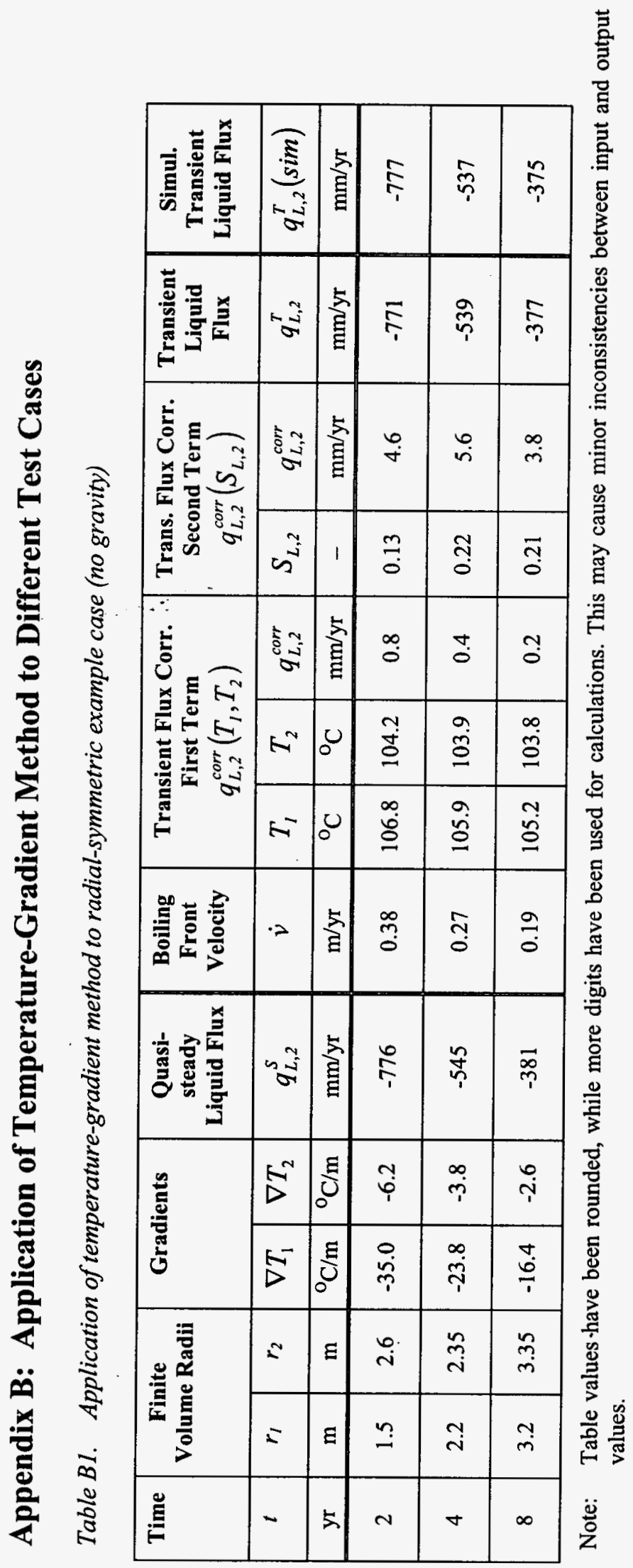




\begin{tabular}{|c|c|c|c|c|c|c|c|c|c|c|c|}
\hline 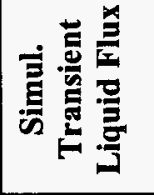 & $\frac{\substack{5 \\
\frac{5}{5}}}{3}$ & है & $\overrightarrow{5}$ & ঙ্ & F్ & f & $\ddot{\vartheta}$ & $\frac{5}{1}$ & 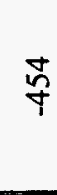 & స్రి & $\bar{\vartheta}$ \\
\hline 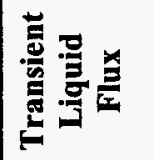 & -3 & ह & $\tilde{n}$ & ఫิ & 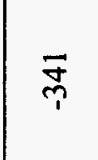 & $\stackrel{m}{n}$ & 礻 & $\frac{6}{1}$ & $\stackrel{\circ}{7}$ & స్ర & $\stackrel{\widetilde{\vartheta}}{\widehat{~}}$ \\
\hline 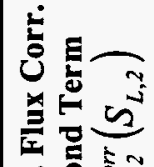 & 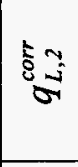 & 占 & $\stackrel{\sim}{\simeq}$ & $\stackrel{\nabla}{\circ}$ & $\bar{\forall}$ & 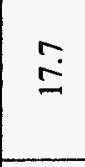 & $\stackrel{\mathfrak{I}}{(}$ & $\ddot{\dot{r}}$ & $\stackrel{m}{i}$ & gे & $\tilde{i}$ \\
\hline 㺼 & $w^{3}$ & 1 & $\frac{5}{0}$ & $\overrightarrow{\tilde{\sigma}}$ & $\frac{9}{0}$ & $\stackrel{\infty}{\rightleftarrows}$ & $\stackrel{\infty}{0}$ & $\stackrel{8}{\circ}$ & $\stackrel{n}{0}$ & $\overline{0}$ & $\stackrel{8}{\circ}$ \\
\hline 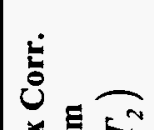 & 童先 & है & $\stackrel{?}{\nabla}$ & $\stackrel{\nabla}{-}$ & $\tilde{0}$ & $\stackrel{\circ}{m}$ & $\stackrel{\nabla}{ت}$ & $\dddot{n}$ & $\stackrel{\wp}{\forall}$ & $\stackrel{t}{i}$ & $\tilde{0}$ \\
\hline 氞言 & $N^{2}$ & 0 & $\begin{array}{l}0 \\
i\end{array}$ & $\stackrel{9}{\dot{0}}$ & $\tilde{n}$ & ㅁ. & $\stackrel{\circ}{\dot{0}}$ & $\stackrel{+}{\dot{0}}$ & $\ddot{n}$ & $\stackrel{\forall}{\oplus}$ & $\stackrel{\infty}{\infty}$ \\
\hline 胥 & Ni & 0 & $\stackrel{\Xi}{\Xi}$ & $\vec{\infty}$ & $\begin{array}{l}\forall \\
\stackrel{0}{0}\end{array}$ & $\begin{array}{l}0 \\
\ddot{0}\end{array}$ & $\stackrel{+}{0}$ & $\stackrel{\circ}{\grave{0}}$ & $\stackrel{m}{\Xi}$ & $\vec{\infty}$ & $\begin{array}{l}\infty \\
\ddot{0}\end{array}$ \\
\hline 里总总 & $\cdot 2$ & 点 & $\frac{2}{0}$ & $\tilde{n}$ & $\stackrel{\overbrace ָ ̣}{0}$ & $\stackrel{\Xi}{\Delta}$ & $\stackrel{\overbrace{}}{\sigma}$ & ભ̊ & ă & $\stackrel{8}{\circ}$ & $\overline{3}$ \\
\hline 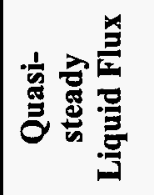 & ำ & 点 & $\stackrel{\infty}{\leftrightarrow}$ & F & 识 & $\frac{\pi}{\frac{\pi}{2}}$ & ஸั & $\underset{T}{T}$ & $\stackrel{?}{7}$ & $\bar{m}$ & ণิ \\
\hline 总 & $\frac{N^{N}}{D}$ & $\frac{E}{0}$ & $\dot{r}$ & $\vec{i}$ & $\stackrel{\circ}{i}$ & $\underset{i ̣}{\dddot{p}}$ & $\stackrel{\eta}{i}$ & $\stackrel{\circ}{i}$ & $\stackrel{0}{r}$ & $\stackrel{a}{i}$ & $\stackrel{n}{i}$ \\
\hline Uूँ & $\frac{B}{D}$ & $\frac{\xi}{0}$ & 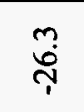 & $\stackrel{n}{\pi}$ & $\stackrel{r}{\square}$ & $\stackrel{n}{7}$ & $\stackrel{r}{\bar{T}}$ & $\underset{\infty}{\sim}$ & กุ & mุ & $\stackrel{\infty}{\circ}$ \\
\hline : & $=2$ & $\Xi$ & $\stackrel{\circ}{\forall}$ & $\stackrel{\vec{n}}{\dot{n}}$ & $\stackrel{\circ}{\sim}$ & $\stackrel{\circ}{\sim}$ & $\begin{array}{l}0 \\
0\end{array}$ & $\vec{a}$ & $\stackrel{\text { テ }}{\sim}$ & $\stackrel{\infty}{\sim}$ & 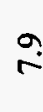 \\
\hline$\frac{1}{3}$ & $=$ & I & $\stackrel{\rho}{\dot{m}}$ & $\stackrel{\circ}{\dot{v}}$ & $\underset{0}{0}$ & $\stackrel{\sim}{q}$ & ஸุ & $\underset{\infty}{\infty}$ & $\stackrel{\infty}{\dot{m}}$ & $\ddot{i}$ & $\stackrel{\circ}{\sim}$ \\
\hline 葛 & - & 5 & $N$ & $\nabla$ & $\infty$ & $N$ & $\theta$ & $\infty$ & $N$ & $\nabla$ & $\infty$ \\
\hline 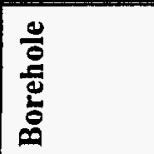 & & & 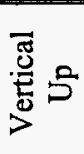 & 莺号 & 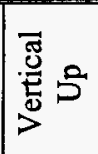 & 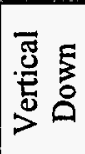 & 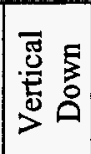 & $\begin{array}{l}\text { 苞 } \\
\text { 志 } \\
\end{array}$ & $\stackrel{\text { N }}{\text { D }}$ & 音 & : \\
\hline
\end{tabular}




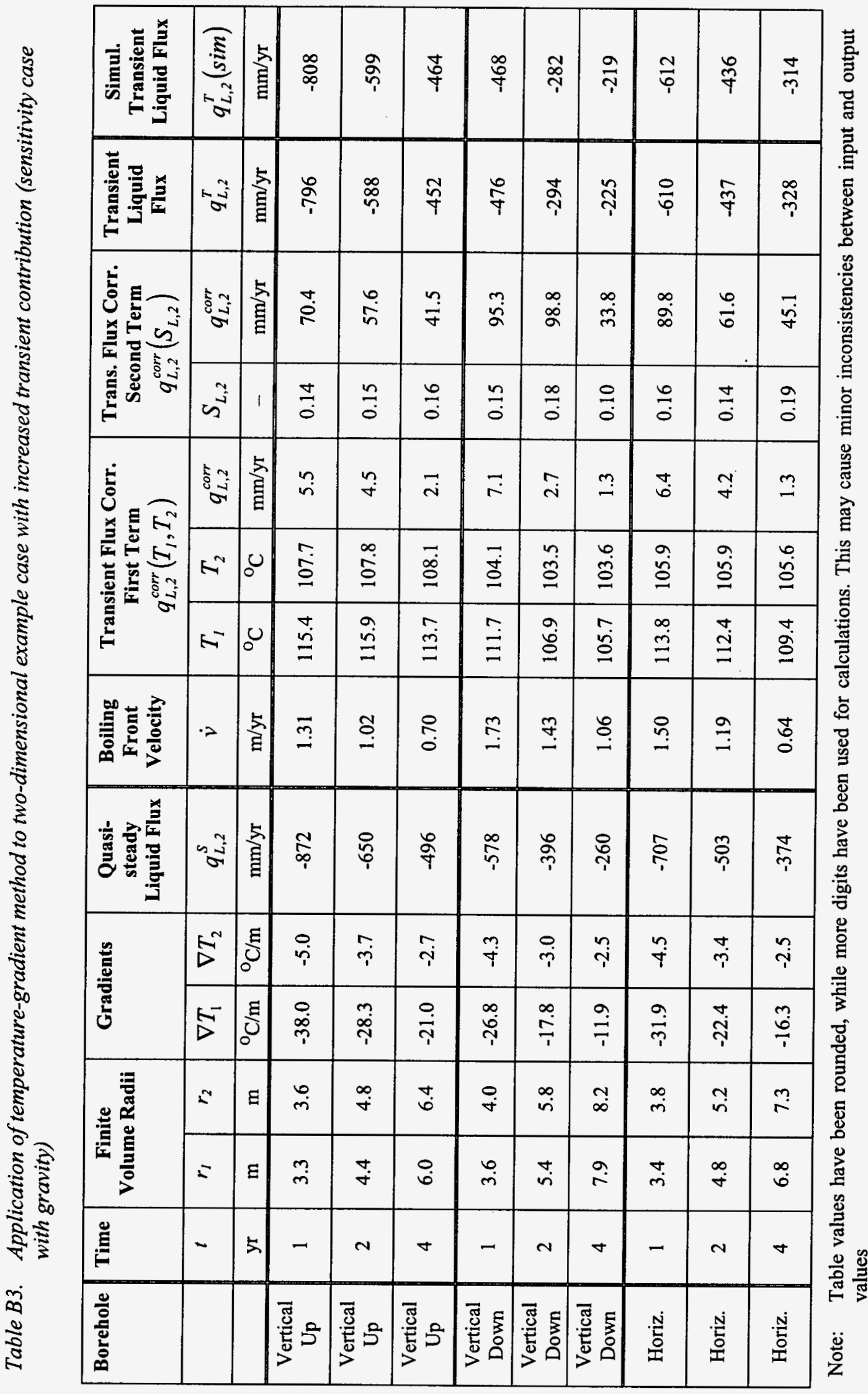




\section{Notation}

A Area, $\mathrm{m}^{2}$

C Specific heat capacity, $\mathrm{J} /(\mathrm{kg}-\mathrm{K})$

$D^{0} \quad$ Binary diffusion coefficient, $\mathrm{m}^{2} / \mathrm{s}$

$h \quad$ Specific enthalpy, $\mathrm{J} / \mathrm{kg}$

$k \quad$ Permeability, $\mathrm{m}^{2}$

$k_{r} \quad$ Relative permeability

$m \quad$ Parameter in van Genuchten characteristic curves

$P \quad$ Pressure, $\mathrm{Pa}$

$P_{c} \quad$ Capillary pressure, $\mathrm{Pa}$

$Q \quad$ Mass flow rate, $\mathrm{kg} /(\mathrm{s}-\mathrm{m})$ for radial geometry, $\mathrm{kg} /\left(\mathrm{s}-\mathrm{m}^{2}\right)$ for linear geometry

$q \quad$ Flux, $\mathrm{m} / \mathrm{s}$

$r \quad$ Radial distance, $\mathrm{m}$

$S \quad$ Saturation

$S_{L r} \quad$ Residual liquid saturation

$T \quad$ Temperature, ${ }^{\circ} \mathrm{C}$

$t \quad$ Time, s

$\dot{v} \quad$ Boiling front velocity, $\mathrm{m} / \mathrm{s}$

$u \quad$ Internal energy, $\mathrm{J} / \mathrm{kg}$

$1 / \alpha \quad$ Parameter in van Genuchten characteristic curves, $\mathrm{Pa}$

$\phi \quad$ Porosity

$\theta$ temperature exponent in binary diffusion equation

$\lambda$ Thermal conductivity, $\mathrm{W} /(\mathrm{m}-\mathrm{K})$ 
$\tau \quad$ Tortuosity

$\rho \quad$ Density, $\mathrm{kg} / \mathrm{m}^{3}$

\section{Subscripts}

G Gas phase

$L \quad$ Liquid phase

$S \quad$ Solid phase

1,2 Relating to inner and outer boundary of finite volume

\section{Superscripts}

$S \quad$ Quasi-steady

$T \quad$ Transient

corr Correction for transient storage terms 


\section{Acknowledgments}

This work was supported by the Director, Office of Civilian Radioactive Waste Management, U.S. Department of Energy, through Memorandum Purchase Order QA-B004220RB3X between Bechtel SAIC Company, LLC and the Ernest Orlando Lawrence Berkeley National Laboratory (Berkeley Lab). The support is provided to Berkeley Lab through the U.S. Department of Energy Contract No. DE-AC0376SF00098. Review and comments of Christine Doughty and Dan Hawkes from Berkeley Lab are gratefully appreciated. We also like to acknowledge the helpful comments of ?? anonymous reviewers.

\section{References}

Bechtel SAIC Company, Drift-scale coupled processes (DST and TH Seepage) models, MDL-NBS-HS-000015 REV 01, Bechtel SAIC Company, Las Vegas, Nevada, 2004a.

Bechtel SAIC Company, Thermal testing measurement report, TDR-MGR-HS-000002 REV 00, Bechtel SAIC Company, Las Vegas, Nevada, 2004b.

Bechtel SAIC Company, Thermal conductivity of the potential repository horizon, MDL-NBS-GS-000005 REV 01, Bechtel SAIC Company, Las Vegas, Nevada, 2004c.

Birkholzer, J.T. and Y.W. Tsang, Modeling the thermal-hydrologic processes in a largescale underground heater test in partially saturated fractured tuff, Water Resour. Res., 36(6), 1431-1447, 2000. 
Birkholzer, J.T. and C.K. Ho, A probabilistic analysis of episodic preferential flow into superheated fractured rock, J. of Hydrology, 284, 151-173, 2003.

Birkholzer, J.T., S. Mukhopadhyay and Y.W. Tsang, Modeling seepage into heated waste emplacement tunnels in unsaturated fractured rock, Vadose Zone J., 3, 819-836, 2004.

Bredehoeft, J.D. and I.S. Papadopolous, Rates of vertical groundwater movement estimated from the earth's thermal profile, Water Resour. Res., 1(2), 325-328, 1965.

Constantz, J., S.W. Tyler and E. Kwicklis, Temperature-profile methods for estimating percolation rates in arid environments, Vadose Zone J., 2, 12-24, 2003.

Datta, R., D. Barr and W. Boyle, Measuring thermal, hydrological, mechanical, and chemical responses in the Yucca Mountain Drift Scale Test, in: Stephansson, Hudson, Jing, editors, Coupled THMC Processes in Geo-Systems: Fundamentals, Modelling, Experiments \& Applications, Elsevier Geo-Engineering Book Series, Oxford, (in press), 155-160, 2004.

Doughty, C. and K. Pruess, A similarity solution for two-phase fluid and heat flow near high-level nuclear waste packages emplaced in porous media, Int. J. Heat Mass Transfer, 33(6), 1205-1222, 1990.

Doughty, C. and K. Pruess, A similarity solution for two-phase water, air, and heat flow near a linear heat source in a porous medium, J. of Geophysical Res., 97(B2), 18211838, 1992. 
Pruess, K., A quantitative model of vapor dominated geothermal reservoirs as heat pipes in fractured porous rock, Transactions, Geothermal Resources Council, 9(ii), 353$361,1985$.

Pruess, K., J.S.Y. Wang and Y.W. Tsang, On thermohydrologic conditions near highlevel nuclear wastes emplaced in partially saturated fractured tuff, 1 , Simulation studies with explicit consideration of fracture effects, Water Resour. Res., 26, 1235 $1248,1990 a$

Pruess, K., J.S.Y. Wang and Y.W. Tsang, On thermohydrologic conditions near highlevel nuclear wastes emplaced in partially saturated fractured tuff, 1, Effective continuum approximations, Water Resour. Res., 26, 1249-1261, $1990 \mathrm{~b}$.

Pruess, K., Oldenburg, K. and G. Moridis, TOUGH2 user's guide, Version 2.0, Rep. LBL-43134 Lawrence Berkeley Natl. Lab., Berkeley, Calif., 1999.

Udell, K.S., Heat transfer in porous media considering phase change and capillarity-the heat pipe effect, Int. J. Heat Mass Tranfer, 28(2), 485-495, 1985.

van Genuchten, M.T., A closed-form equation for predicting the hydraulic conductivity of unsaturated soils, Soil Science Society of America Journal, 44, (5), 892-898, 1980. 
FIGURES

$-45-$ 


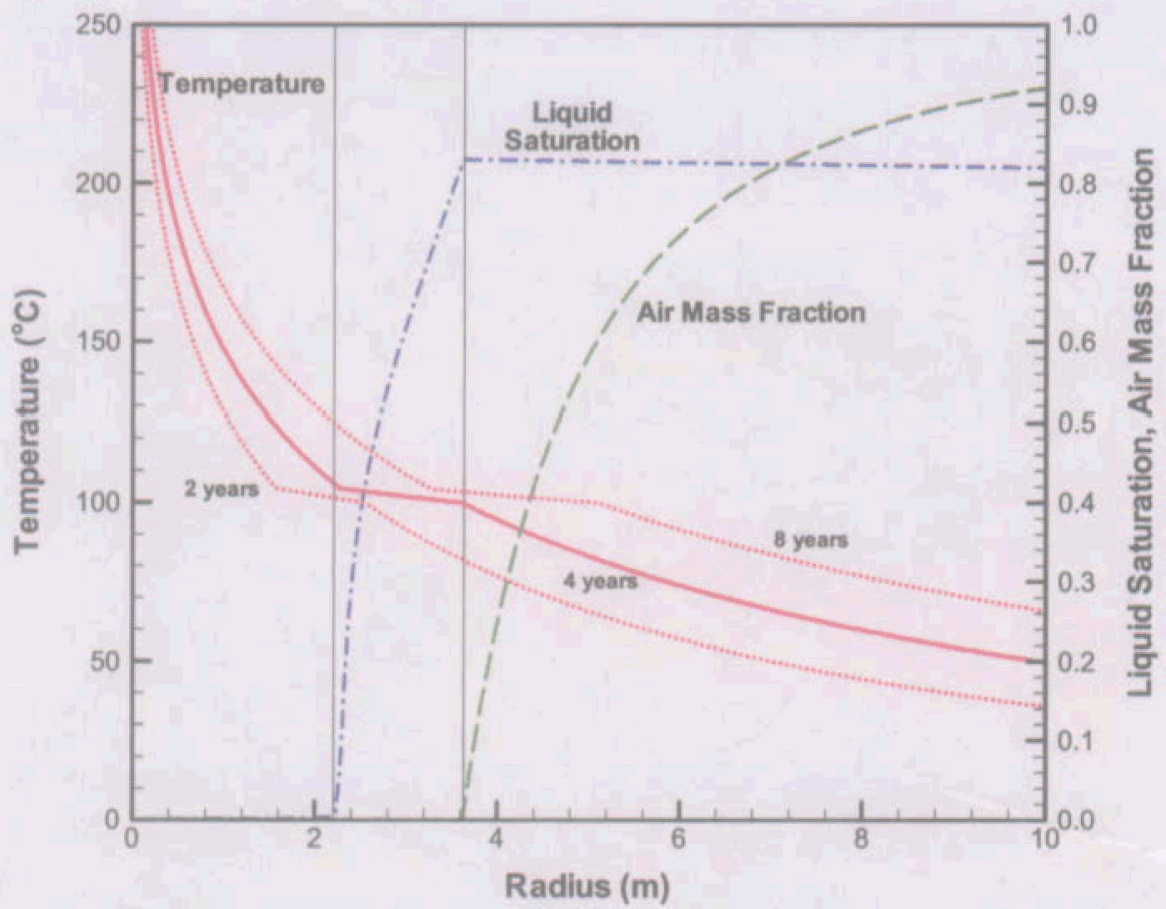

(a)

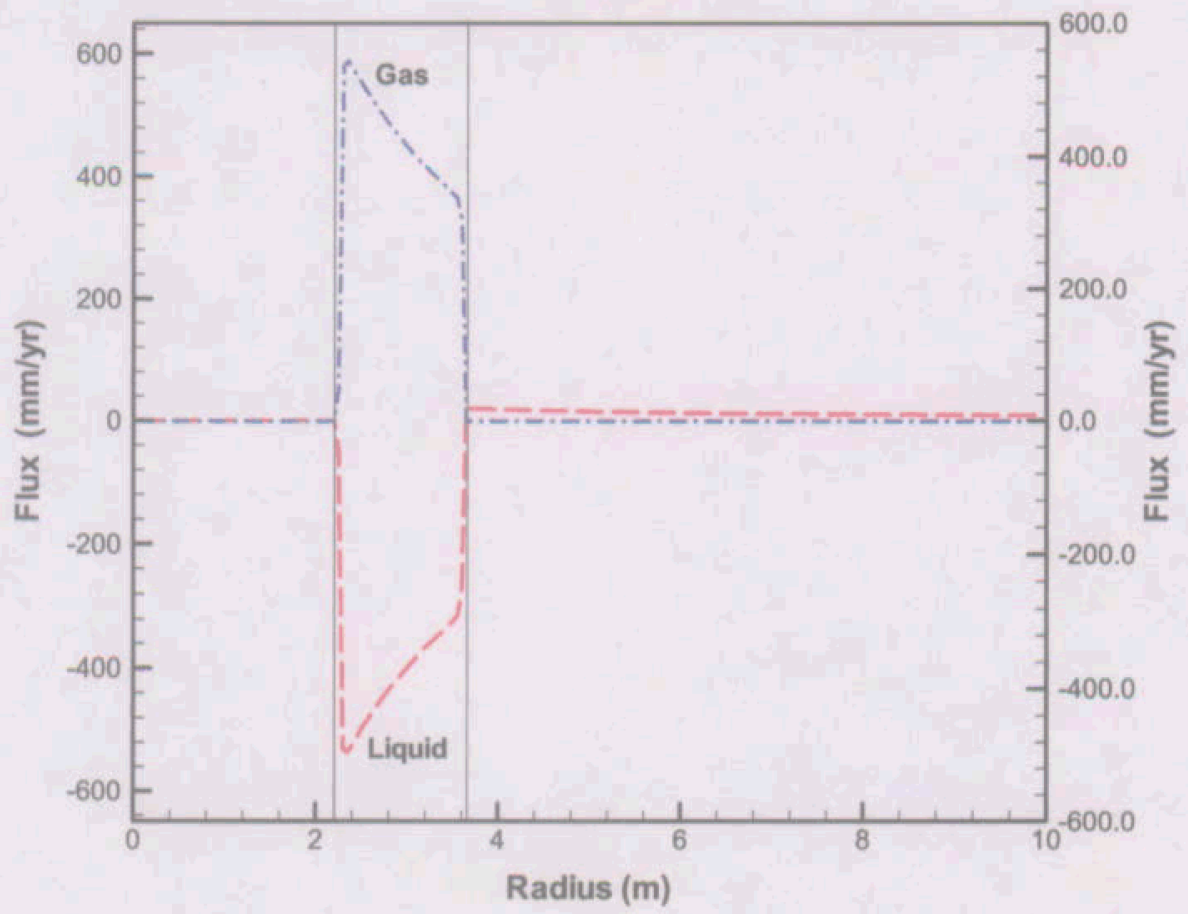

(b)

Figure 1. Results for the radial-symmetric heat pipe system (without gravity) studied in Doughty et al. (1992), showing profiles at 4 years for (a) temperature, liquid saturation, and air mass fraction, as well as (b) gas and liquid flux. For comparison, additional temperature profiles are depicted at 2 years and 8 years. 


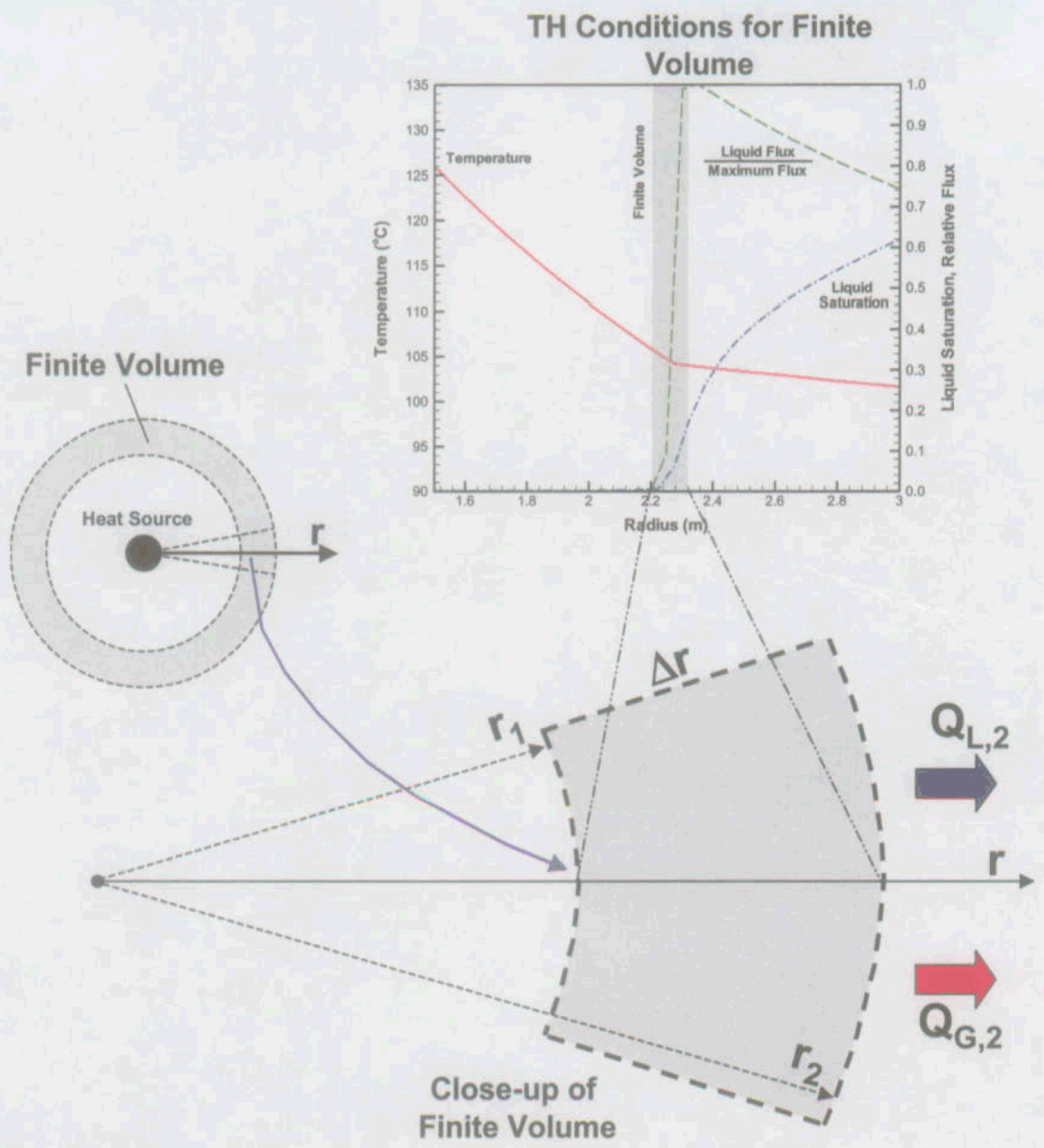

Figure 2. Definition of finite volume for temperature-gradient method 


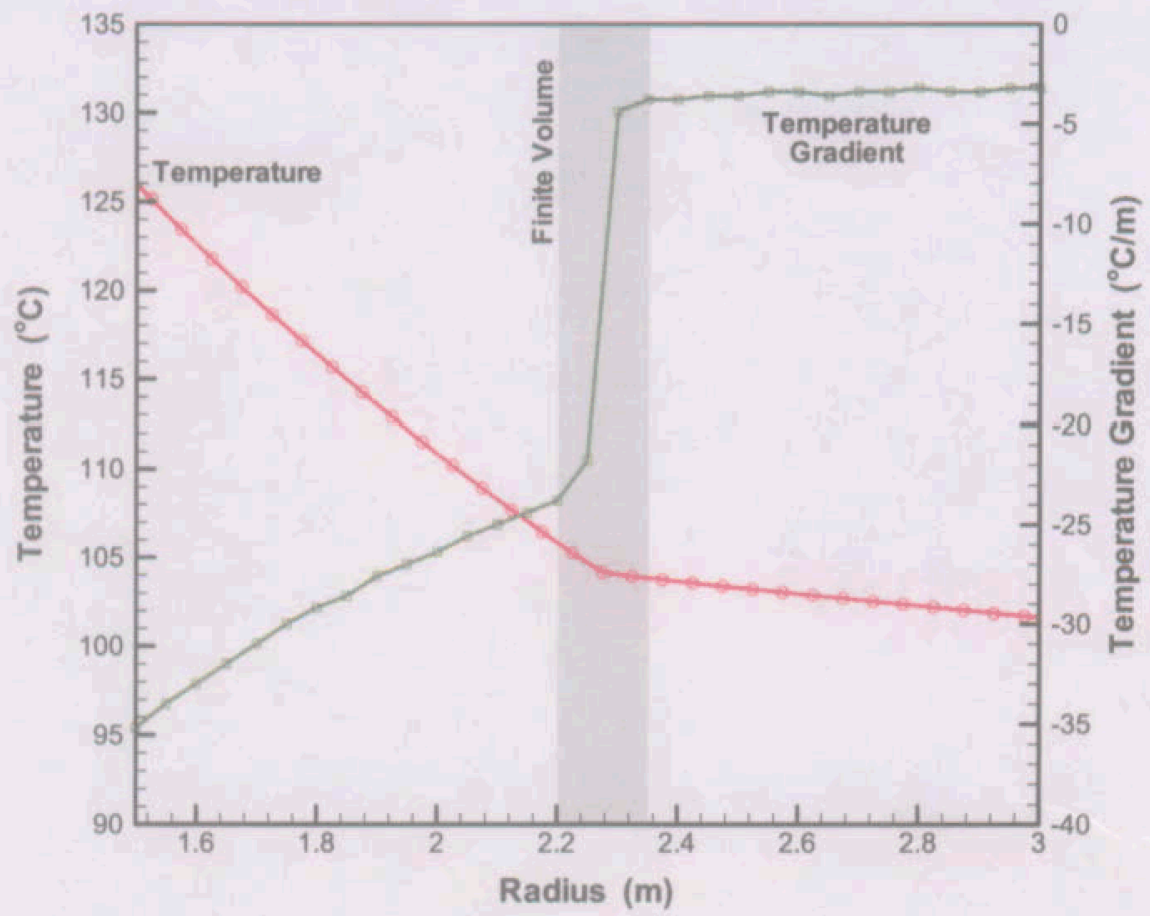

Figure 3. Temperature-gradient method for the radial-symmetric heat pipe system (without gravity) studied in Doughty and Pruess (1992) and Pruess et al. (1999), giving results at 4 years. Plot shows simulated temperature at nodal points, average gradient between two nodal points, and choice of finite volume. 


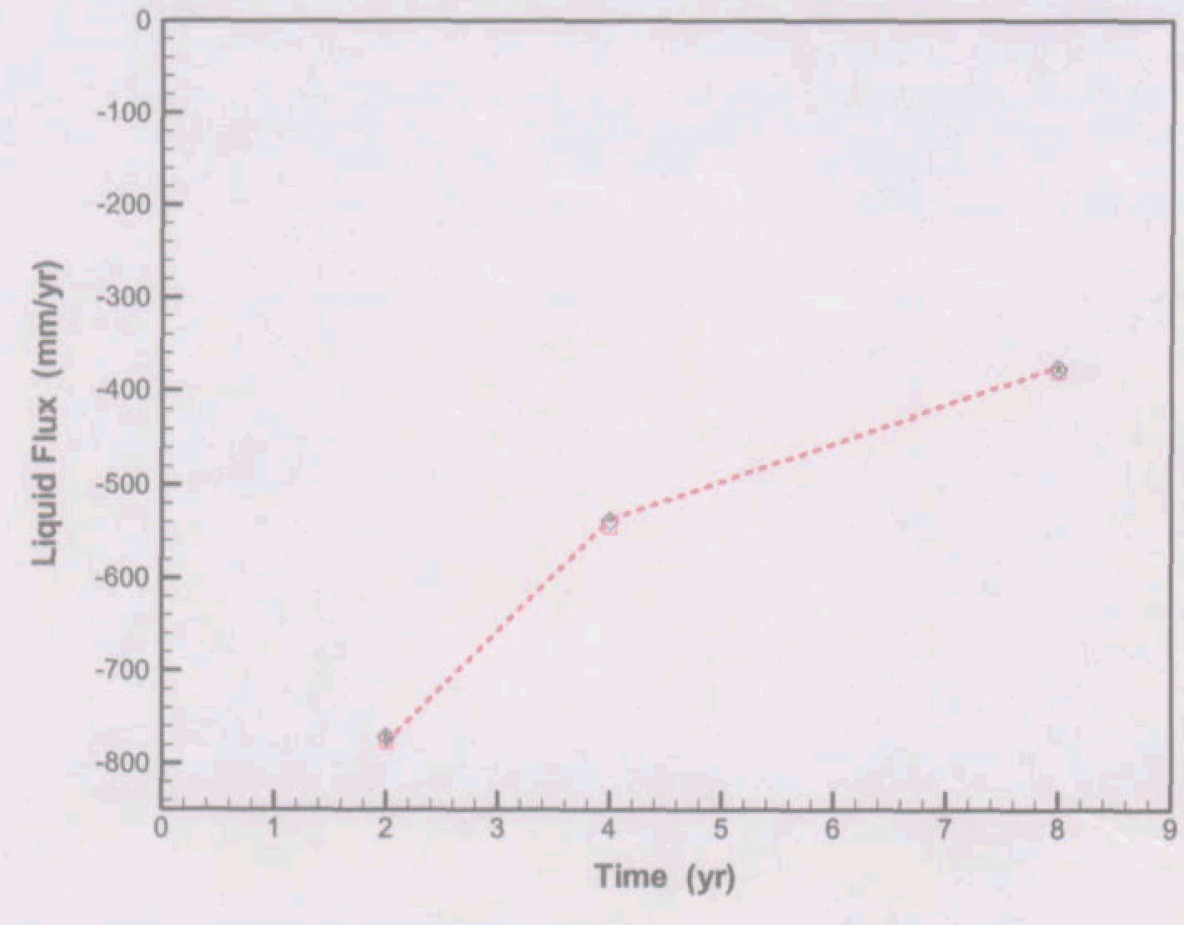

Figure 4. Fhax estimates from temperature-gradient method for radial-symmetric heat pipe system in comparison with simulated fluxes. Squares give estimated fluxes using the quasi-steady heat pipe solution. Diamonds give estimated fluxes assuming transient heat pipes. Small triangles show transient solution fluxes for four additional upstream temperature gradients. Dashed line connects simulated flux values. 

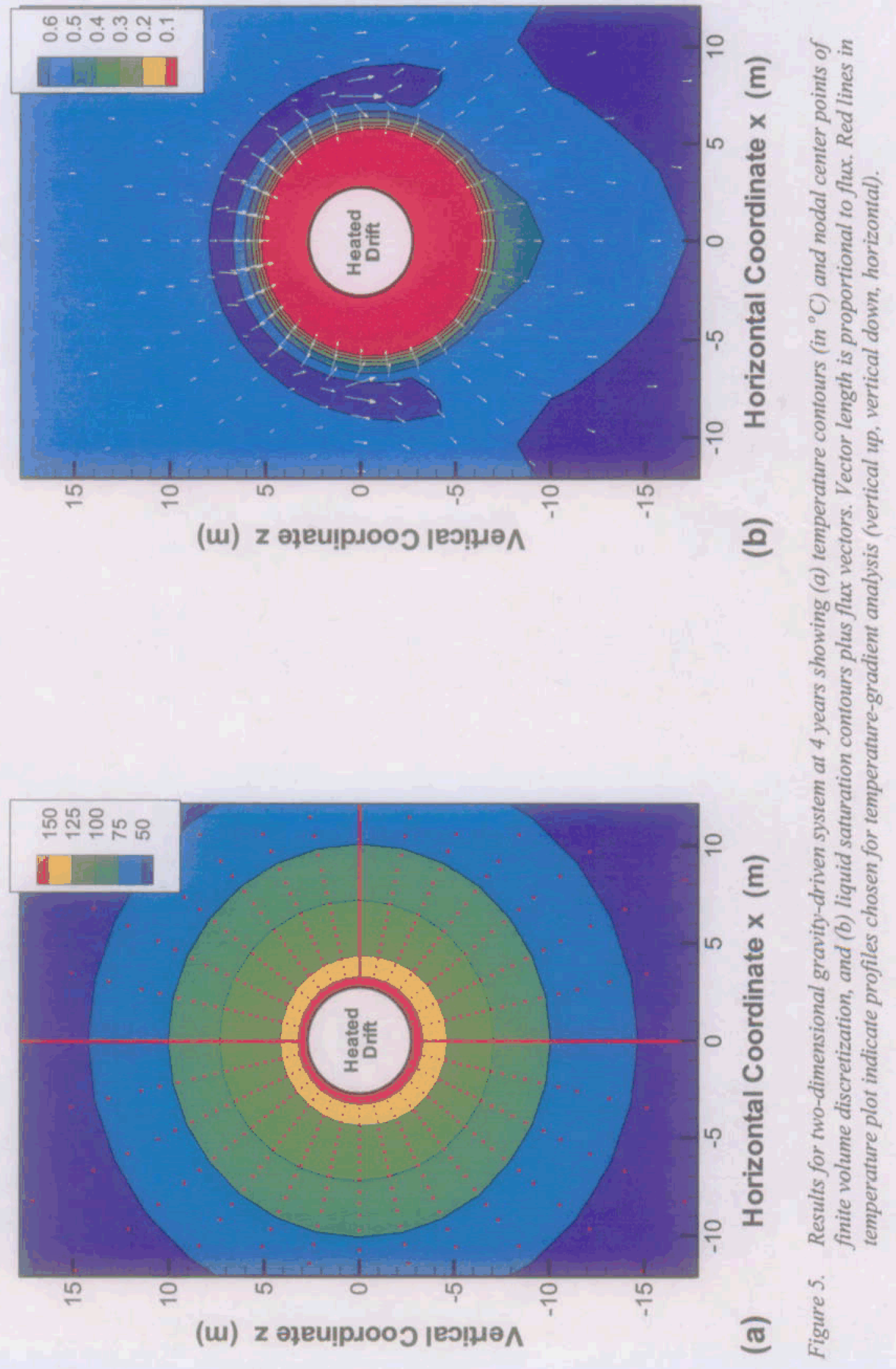


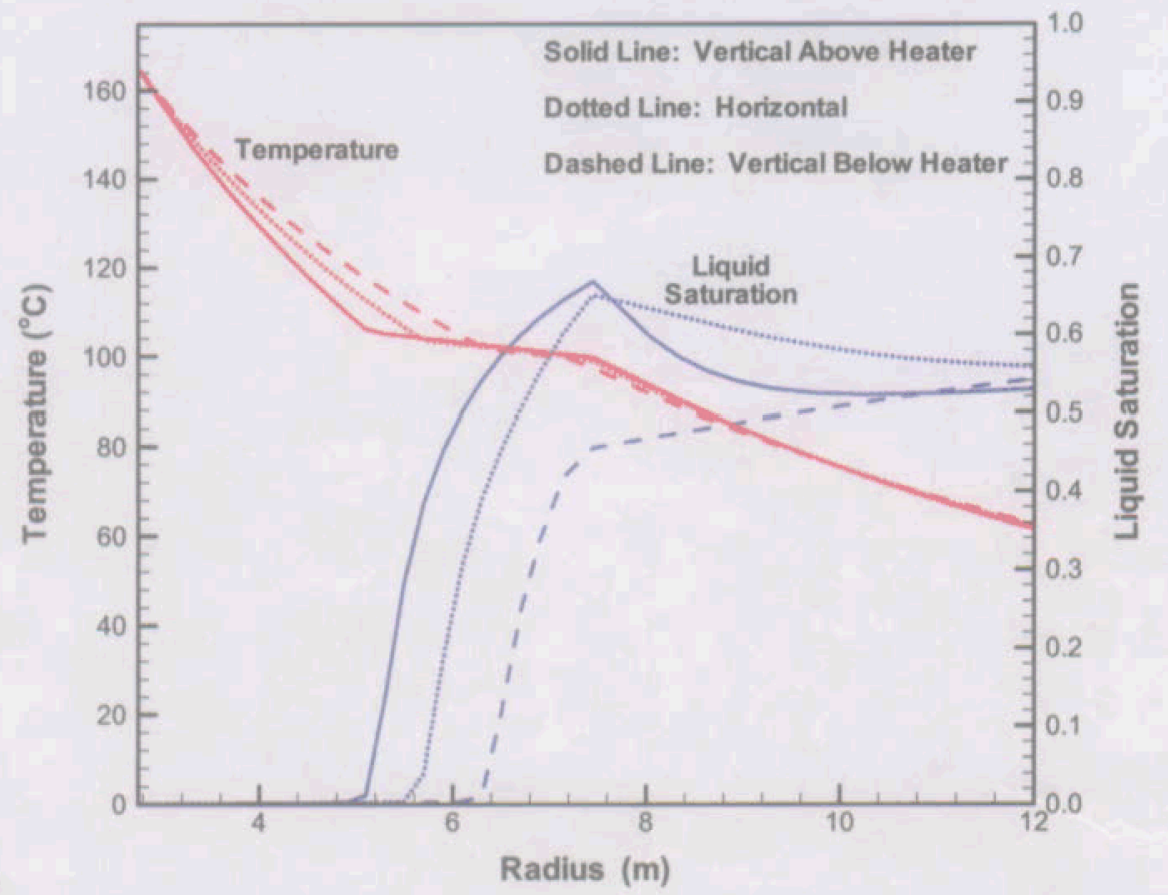

(a)

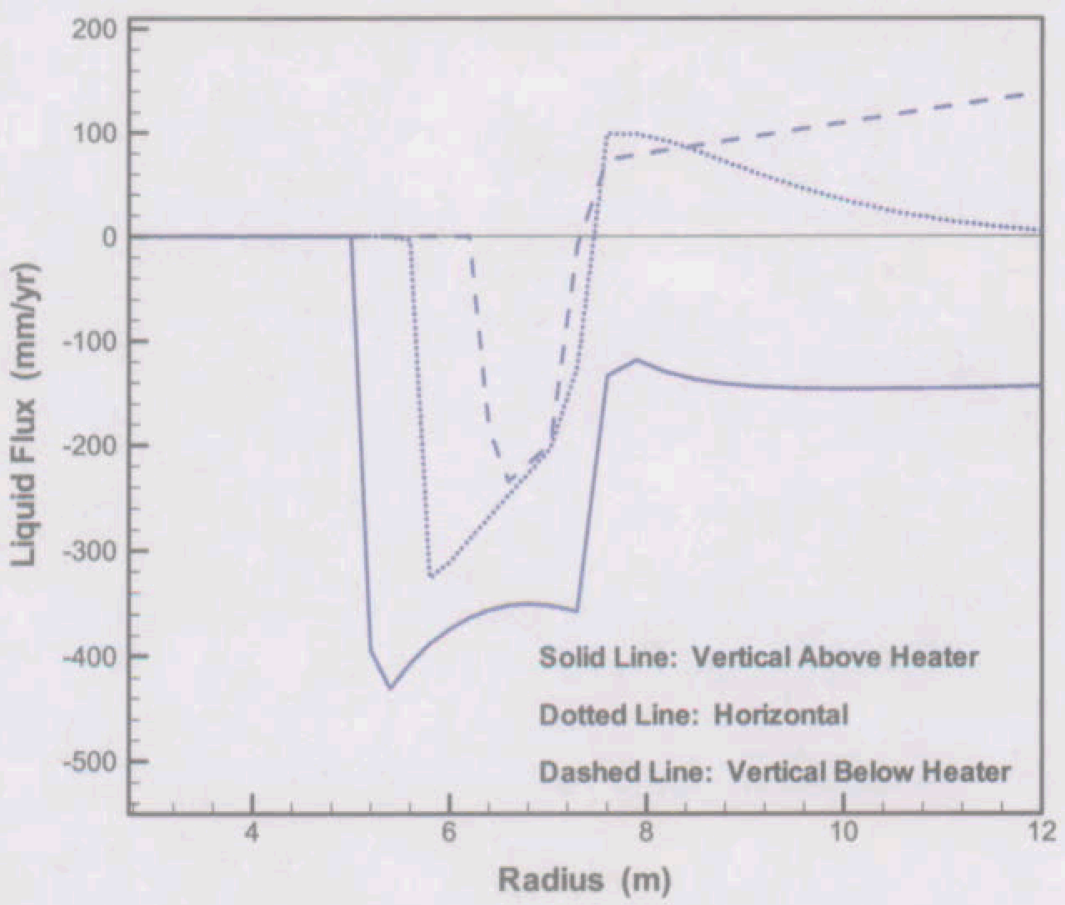

Figure 6. Results for two-dimensional gravity-driven system (base case) at 4 years showing (a) temperature and saturation profiles, as well as (b) liquid flux profiles. Selected profiles are vertical above and below the heater at $x=0 \mathrm{~m}$, and horizontal in positive $x$-direction at $z=0 \mathrm{~m}$. For better comparison, profiles are given as a function of radial distance rather than coordinate. Flux convention as follows: Positive fluxes moving outward, away from heat source. Negative fluxes back towards heat source. 


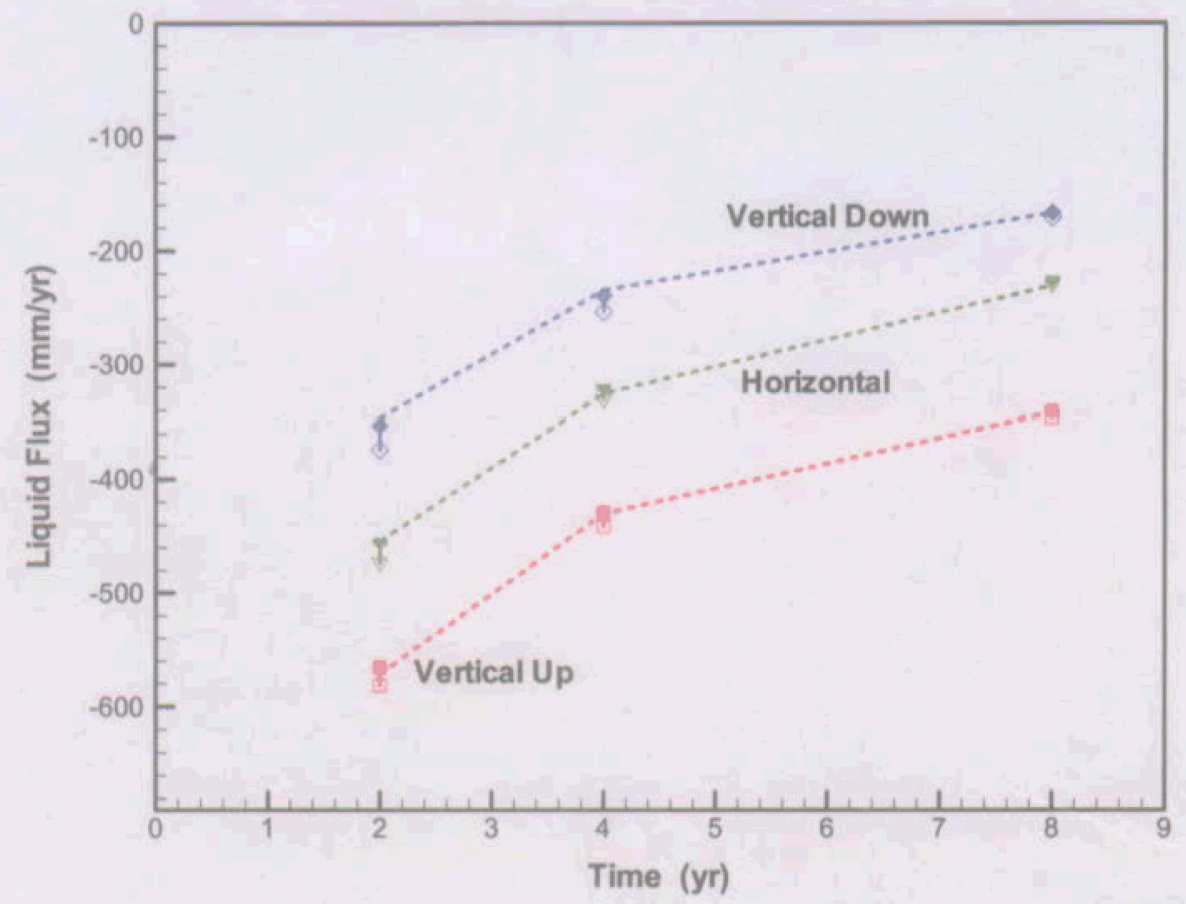

Figure 7. Flux estimates from temperature-gradient method for two-dimensional gravity-driven system (base case) in comparison with simulated fluxes. Hollow symbols give estimated fluxes using the quasi-steady heat pipe solution. Solid symbols give estimated fluxes assuming transient heat pipes. Dashed lines connect simulated flux values. 


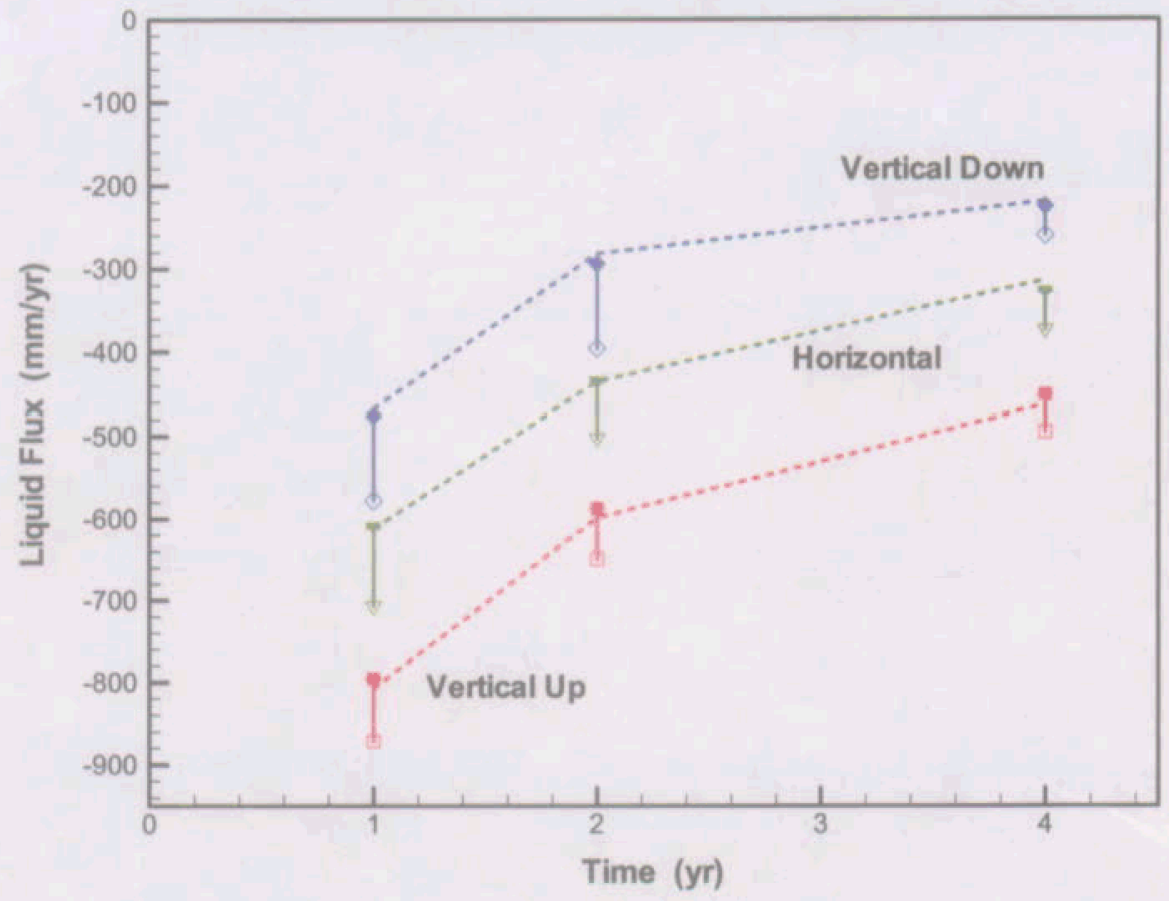

Figure 8. Flux estimates from temperature-gradient method for two-dimensional gravity-driven system (sensitivity case with stronger heat source and larger porosity) in comparison with simulated fluxes. Hollow symbols give estimated fluxes using the quasi-steady heat-pipe solution. Solid symbols give estimated fluxes assuming transient heat pipes. Dashed lines connect simulated flux values. 

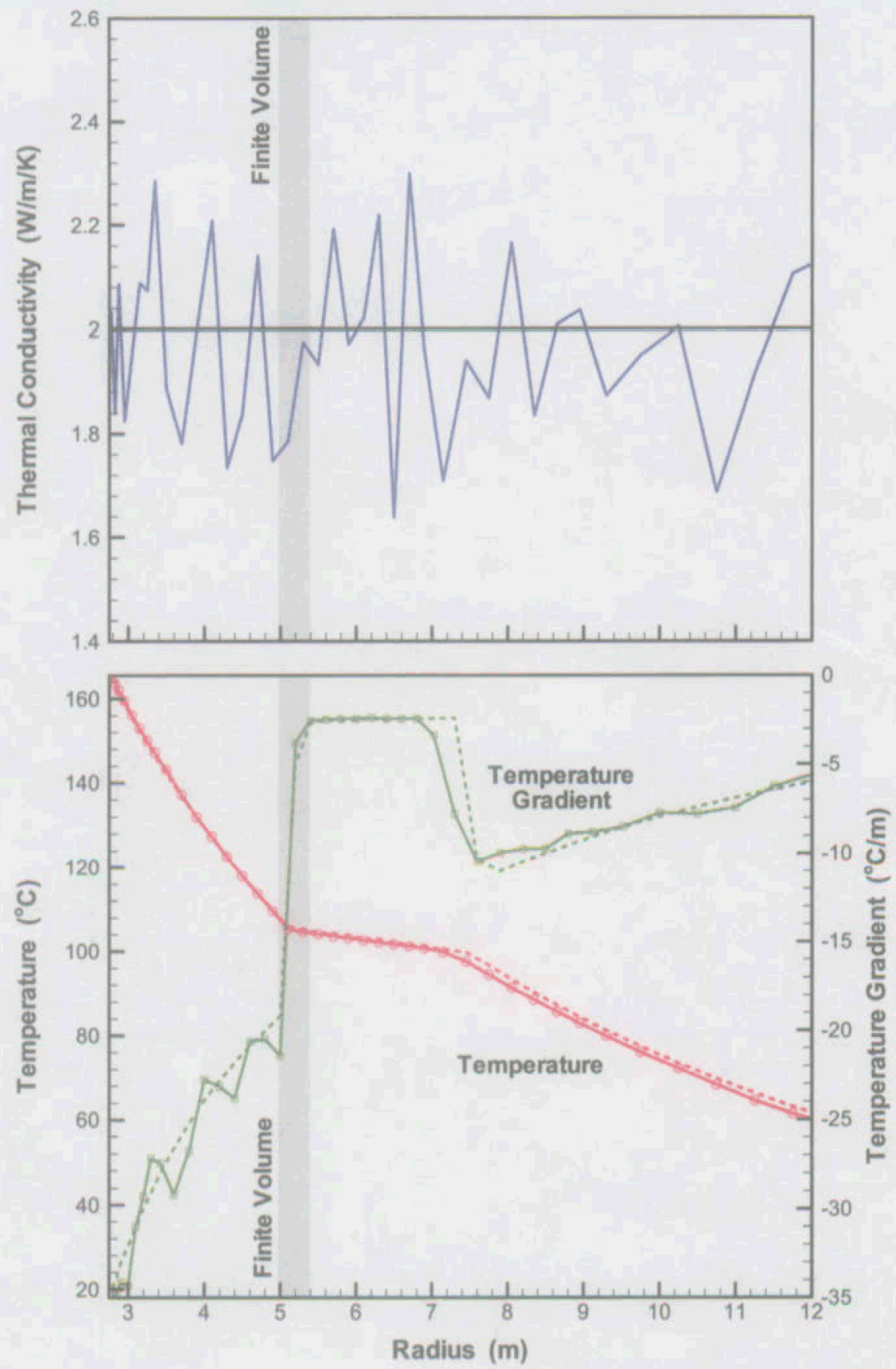

Figure 9a. Results for two-dimensional gravity-driven system at 4 years in borehole vertically upward. Upper plot shows distribution of thermal conductivity. Lower plot shows simulated temperature at nodal points (given in radial distance from drift center), average gradient between two sensors, and choice of finite volume for temperaturegradient method. Solid lines are for heterogeneous sensitivity case. Dashed lines show base case results for comparison. 

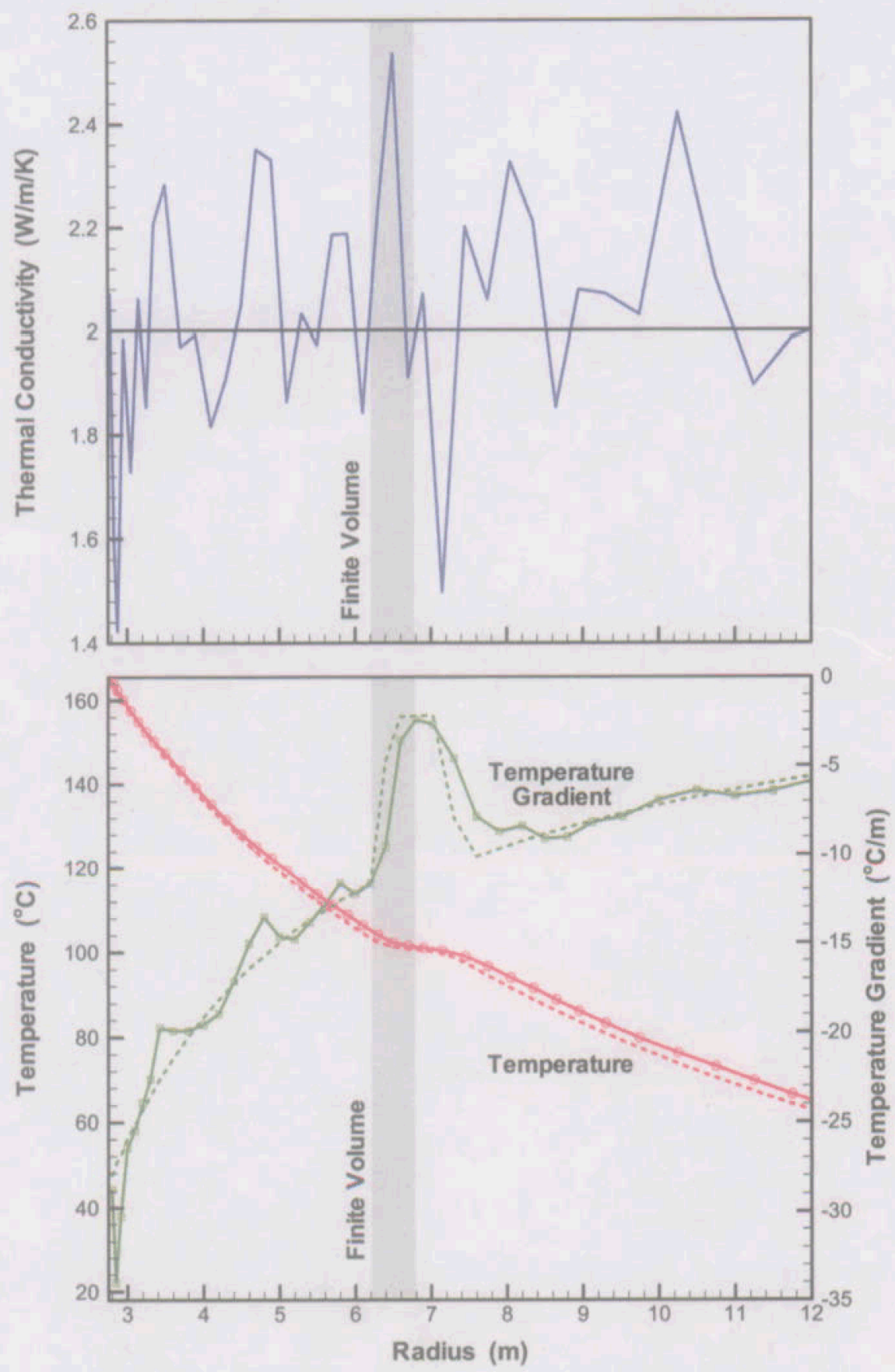

Figure $9 b$. Results for two-dimensional gravity-driven system at 4 years in borehole vertically downward. Upper plot shows distribution of thermal conductivity. Lower plot shows simulated temperature at nodal points (given in radial distance from drift center), average gradient between two sensors, and choice of finite volume for temperaturegradient method. Solid lines are for heterogeneous sensitivity case. Dashed lines show base case results for comparison. 

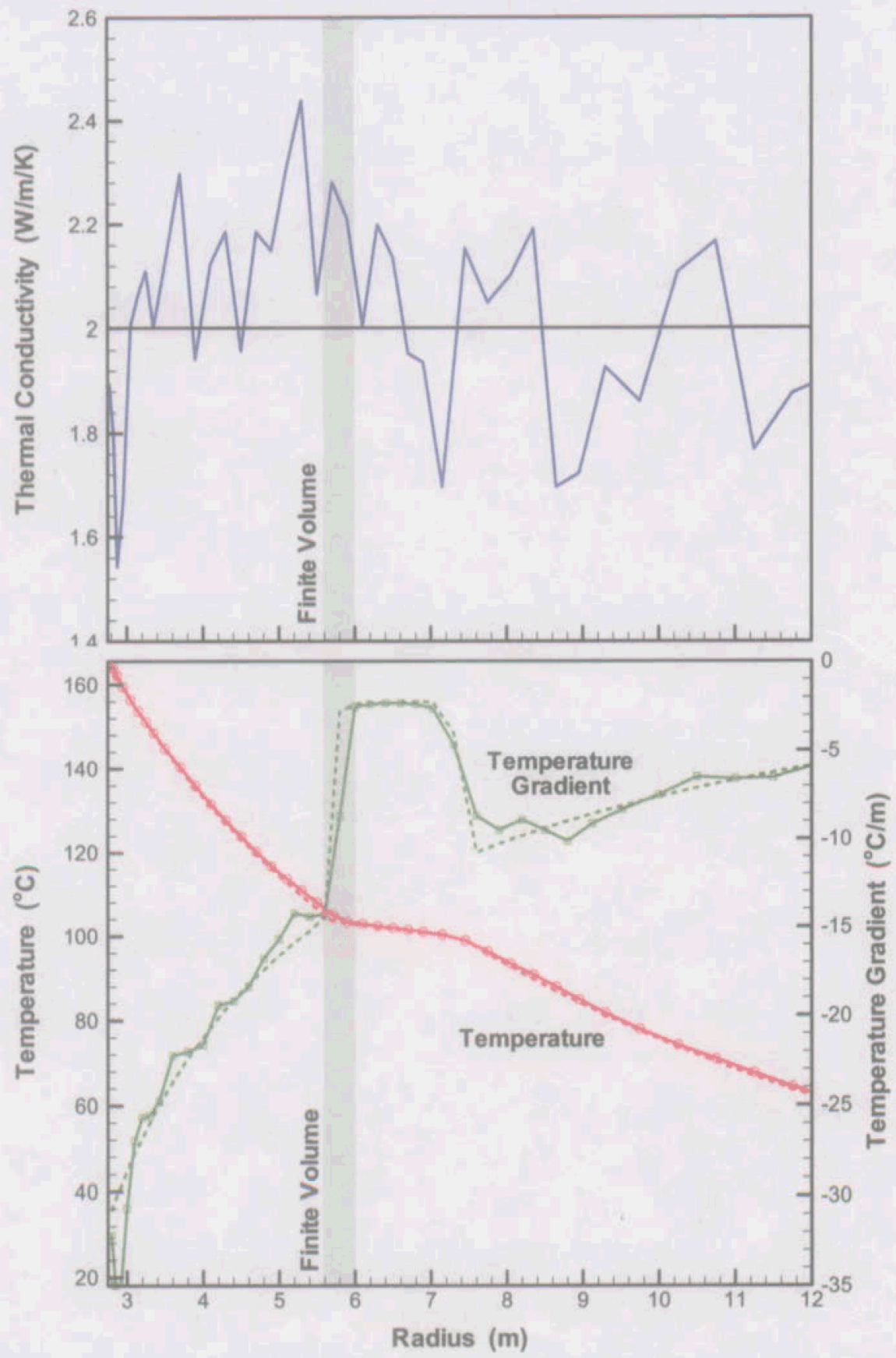

Figure $9 c$. Results for two-dimensional gravity-driven system at 4 years for horizontal borehole. Upper plot shows distribution of thermal conductivity. Lower plot shows simulated temperature at nodal points (given in radial distance from drift center), average gradient between two sensors, and choice of finite volume for temperature-gradient method. Solid lines are for heterogeneous sensitivity case. Dashed lines show base case results for comparison. 


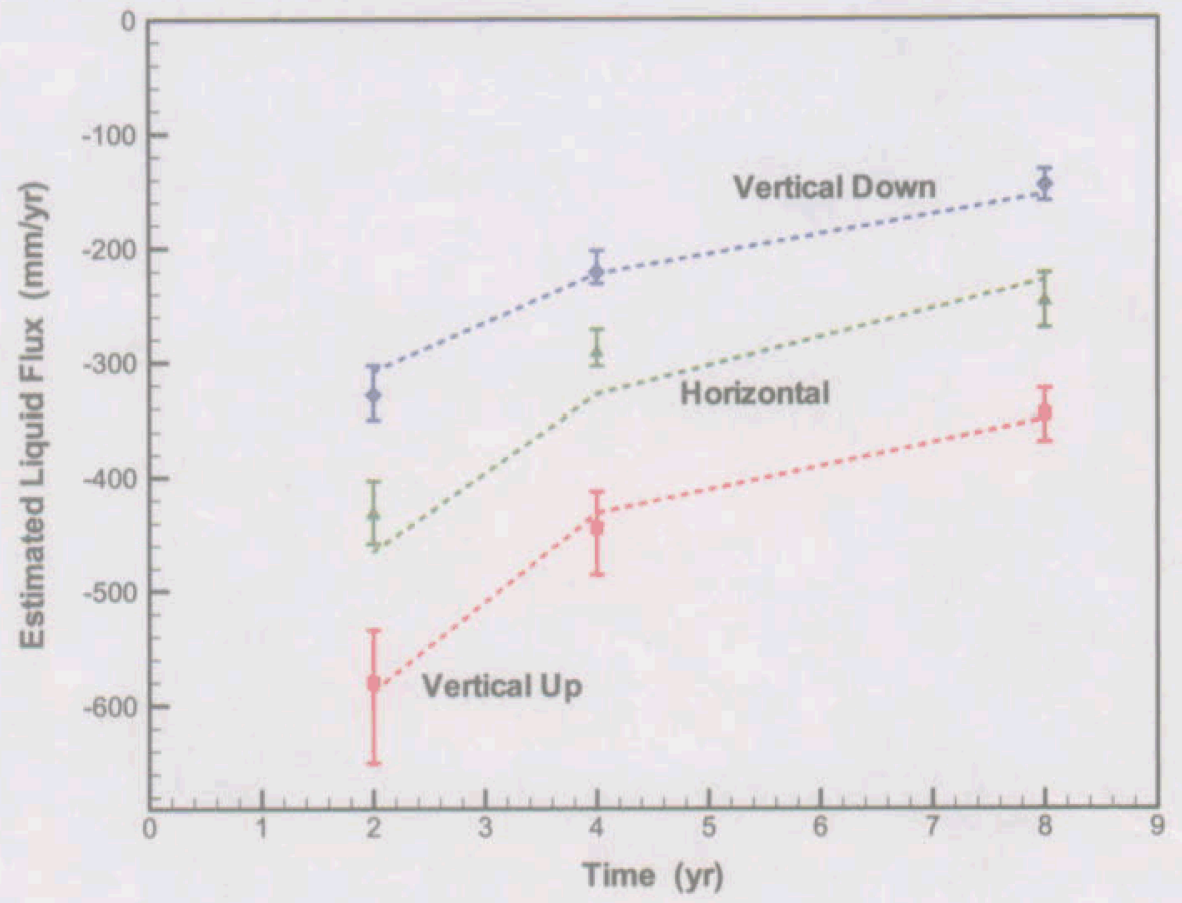

Figure 10. Flux estimates from temperature-gradient method for two-dimensional gravity-driven system (heterogeneous sensitivity case) in comparison with simulated fluxes. Solid symbols give estimated fluxes assuming transient heat pipes. Error bars indicate range of five individual flux estimates. Dashed lines connect simulated flux values. 
TABLES

- 58 - 
Table 1. Parameters for test problems evaluated in Sections 3 and 4

Parameter Value

Initial Conditions

Pressure $P$

Temperature $T$

Saturation $S_{L}$
1 bar

$18^{\circ} \mathrm{C}$

0.8 for Section 3, 0.6 for Section 4

Material Properties of Porous Medium

Permeability $k$

Porosity $\phi$

Grain density $\rho_{S}$

Grain heat capacity $C_{S}$

Thermal conductivity $\lambda$

Tortuosity $\tau$

Binary diffusion coefficient $D^{0}$

Temperature exponent $\theta$

\section{Characteristic Curves of Porous Medium}

Capillary pressure, $P_{c}$

Relative Permeability liquid, $k_{r L}$

Relative Permeability gas, $k_{r G}$

Van Genuchten parameter $S^{*}$

Residual liquid saturation $S_{L r}$

Van Genuchten parameter $l / \alpha$

Van Genuchten parameter $m \quad 0.45$

Maximum capillary pressure $P_{\max }$

$$
\begin{aligned}
& 2 \times 10^{-14} \mathrm{~m}^{2} \\
& 0.10(0.4 \mathrm{in} \text { sensitivity case }) \\
& 2550 \mathrm{~kg} / \mathrm{m}^{3} \\
& 800 \mathrm{~J} / \mathrm{kg} / \mathrm{K} \\
& 2 \mathrm{~W} / \mathrm{m} / \mathrm{K} \\
& 0.25 \\
& 2.6 \times 10^{-5} \mathrm{~m}^{2} / \mathrm{s} \\
& 1.8
\end{aligned}
$$

$$
-1 / \alpha\left[\left(S^{*}\right)^{-1 / m}-1\right]^{1-m}
$$$$
\left(S^{*}\right)^{1 / 2}\left[1-\left(1-\left(S^{*}\right)^{1 / m}\right)^{m}\right]^{2}
$$

$$
l-k_{r L}
$$$$
\left(S_{L}-S_{L r}\right) /\left(1-S_{L r}\right)
$$$$
9.6 \times 10^{-4}
$$

$$
0.125 \text { bar }
$$

5000 bar

Thermodynamic Properties of Water and Vapor (at $P=1$ bar and $T=99.6^{\circ} \mathrm{C}$ )

Density of water $\rho_{L}$

Specific heat of vaporization $h_{G}-h_{L}$
$958 \mathrm{~kg} / \mathrm{m}^{3}$

$2.26 \times 10^{6} \mathrm{~J} / \mathrm{kg}$

Note: The governing equations of the two-phase flow and transport processes simulated in the considered test example are described in Doughty and Pruess (1992). The characteristic curves utilize the functional forms introduced by van Genuchten (1980), with a slight modification regarding the maximum possible capillary pressure (Doughty and Pruess, 1992). The chosen binary diffusion properties (i.e., tortuosity, diffusion coefficient, temperature exponent) are identical to Problem No. 2 in Pruess et al. (1999), which differ slightly from those in Doughty and Pruess (1992). 